\title{
The endocrine tumor summit 2008: appraising therapeutic approaches for acromegaly and carcinoid syndrome
}

\author{
Anne Klibanski - Shlomo Melmed • David R. Clemmons • \\ Annamaria Colao - Regina S. Cunningham • Mark E. Molitch • \\ Aaron I. Vinik - Daphne T. Adelman - Karen J. P. Liebert
}

Published online: 13 December 2009

(C) The Author(s) 2009. This article is published with open access at Springerlink.com

\begin{abstract}
The Endocrine Tumor Summit convened in December 2008 to address 6 statements prepared by panel members that reflect important questions in the treatment of acromegaly and carcinoid syndrome. Data pertinent to each of the statements were identified through review of pertinent literature by one of the 9-member panel, enabling a critical evaluation of the statements and the evidence supporting or refuting them. Three statements addressed the validity of serum growth hormone $(\mathrm{GH})$ and insulin-like growth
\end{abstract}

This activity is supported by an educational grant from Ipsen.

\section{A. Klibanski $(\square)$}

Massachusetts General Hospital, Harvard Medical School,

55 Fruit Street, Boston, MA 02114, USA

e-mail: aklibanski@partners.org

\section{S. Melmed}

Cedars-Sinai Medical Center, Academic Affairs, Room \#2015, 8700 Beverly Boulevard, Los Angles, CA 90048, USA

e-mail: Shlomo.melmed@cshs.org

\section{R. Clemmons}

University of North Carolina School of Medicine, 8024 Burnette

Womack, CB 7170, Bowles Building, Chapel Hill,

NC 27599-7170, USA

e-mail: endo@medicineexch.med.unc.edu;

david_clemmons@med.unc.edu

\section{A. Colao}

Department of Molecular and Clinical Endocrinology and Oncology, University Federico II of Naples, Via S. Pansini 5, Naples 80131, Italy

e-mail: colao@unina.it

\section{R. S. Cunningham}

The Cancer Institute of New Jersey, Robert Wood Johnson Medical Center, 195 Little Albany Street, New Brunswick, NJ 08903-2681, USA

e-mail: xlilrbcqtx@yahoo.com
factor-I (IGF-I) concentrations as indicators or predictors of disease in acromegaly. Statements regarding the effects of preoperative somatostatin analog use on pituitary surgical outcomes, their effects on hormone and symptom control in carcinoid syndrome, and the efficacy of extended dosing intervals were reviewed. Panel opinions, based on the level of available scientific evidence, were polled. Finally, their views were compared with those of surveyed communitybased endocrinologists and neurosurgeons.

\section{E. Molitch}

Northwestern University Feinberg School of Medicine, 645 N. Michigan Avenue Suite 530, Chicago, IL 60611, USA

e-mail: molitch@northwestern.edu

\author{
A. I. Vinik \\ Department of Internal Medicine, Eastern Virginia Medical \\ School, Strelitz Diabetes Center, 855 West Brambleton Ave., \\ Norfolk, VA 23510, USA \\ e-mail:VinikAI@evms.edu \\ D. T. Adelman \\ Northwestern University Feinberg School of Medicine, 645 N. \\ Michigan Avenue Suite 530, Chicago, IL 60611, USA \\ e-mail: d-adelman@northwestern.edu \\ K. J. P. Liebert \\ Neuroendocrine Unit, Massachusetts General Hospital, Bulfinch \\ 457 B, 55 Fruit Street, Boston, MA 02114, USA \\ e-mail: kpulaski@partners.org
}


Keywords Acromegaly - Carcinoid syndrome . Neuroendocrine tumor - NET $\cdot$ Somatostatin analogs . Somatostatin analogues - Lanreotide - Octreotide . Insulin-like growth factor-I · IGF-I .

Growth hormone $\cdot \mathrm{GH}$

\section{Introduction}

Despite considerable progress in defining criteria for disease control in patients with acromegaly, a number of controversies exist regarding the use of GH and IGF-I measurements towards this end. Similarly, the use of somatostatin analogs in the treatment of acromegaly has strikingly altered disease outcomes for patients with this disease, as well as symptom control for patients with neuroendocrine tumors (NETs). However, issues of use of these medications preoperatively in patients with acromegaly, dosing intervals, and treatment efficacy remain. In the Summit proceedings summarized, an expert panel was asked to examine a number of controversial statements pertaining to current therapeutic approaches in the management of acromegaly and carcinoid syndrome (Table 1). Evidence both supporting and contradicting the statement was presented, and panelists were asked to decide, based on the evidence in the literature, on their degree of acceptance of each statement. The results from the Summit voting were compared with the findings of an electronic survey containing the same statements as voted on by the workshop panel. The 100 respondents consisted of endocrinologists and neurosurgeons, all of whom treat patients with either acromegaly or neuroendocrine and/or pituitary tumors. Survey respondents have practiced from 1 to 10 years and represented various geographic regions of the United States.

\section{Statement 1: an IGF-I value that is normal for age and gender signifies control of acromegaly}

Rationale and definition of statement

Although serum IGF-I levels offer value in monitoring control of acromegaly, their precise role is still developing. The increasing body of data in this relatively small population warrants a new critical look, comparing it with biochemical markers such as $\mathrm{GH}$ as well as other endpoints used to assess acromegaly control, including mortality, morbidity, symptom control, and quality of life (QOL).

\section{Literature search}

A literature search was undertaken in October 2008 using the search terms "serum IGF-I," "serum somatomedin C," and "diabetes," each combined with "acromegaly." 1121 titles were reviewed; 130 abstracts were selected for further review. Of these, 47 publications that appeared to be relevant were examined more comprehensively, resulting in 15 that were directly relevant to the statement.

\section{Evidence}

One of the principal indices of remission in acromegaly has been the GH response to oral glucose tolerance testing (OGTT). To determine whether there was a relationship between the response of glucose-suppressed GH and serum IGF-I, Vierhapper et al. [1] analyzed 26 untreated acromegalic patients and 71 patients post transsphenoidal surgery. OGTT with GH sampling and IGF-I were measured in all subjects. IGF-I was found to vary with age and

Table 1 Clinical practice statements

\section{Statements $1-6$}

1. An IGF-I value that is normal for age and gender signifies control of acromegaly

2. A GH of $\leq 1.0 \mu \mathrm{g} / \mathrm{l}$ as a random measurement or as a nadir after an oral glucose tolerance test correlates with disease control in patients with acromegaly

3. GH levels sampled at any time interval are better predictors of control of acromegaly and related morbidity than IGF-I levels

4. The administration of somatostatin analogs prior to transsphenoidal surgery improves surgical outcome and disease morbidity associated with acromegaly

5. The dosing interval of a somatostatin analog can be extended beyond once every 4 weeks without compromising disease control in patients with acromegaly

6. A monthly injection of the depot form of lanreotide or octreotide results in consistent control of hormone levels and symptoms in patients with carcinoid syndrome

$I G F-I$ insulin-like growth factor-I, $G H$ growth hormone 
gender, and GH suppression varied with age and BMI. Post surgery, IGF-I response and normalization of glucosesuppressed GH were congruent in $77 \%$ of the patients. The authors demonstrated that across the board cutoff values for either parameter could not be applied to the entire population. They concluded that post-treatment OGTT GH and IGF-I responses should be interpreted individually.

One problem in attempting to correlate IGF-I values and GH suppression following treatment of acromegaly has been to establish cutoff values for GH suppression. Over the years, this cutoff value has varied from $5 \mathrm{ng} / \mathrm{ml}$ to the current recommendation of less than $2.5 \mathrm{ng} / \mathrm{ml}$ by conventional radioimmunoassay or less than $1 \mathrm{ng} / \mathrm{ml}$ by sensitive chemiluminescence assay. The natural history and ultimate outcome of patients with values between 0.3 and $1 \mathrm{ng} / \mathrm{ml}$ has not been widely studied in detail.

Ronchi et al. [2] studied 40 subjects followed for 14.3 years \pm 4.2 (mean $\pm \mathrm{SD}$ ) after transsphenoidal surgery. The authors stratified patients into two levels of GH suppression: $\mathrm{GH}<0.19 \mathrm{ng} / \mathrm{ml}$ (defining a cure) or $\mathrm{GH}<0.77 \mathrm{ng} / \mathrm{ml}$ (defining a major improvement). No difference in clinical outcome was noted between those groups. All subjects in both groups had a normal IGF-I; only $2 \%$ of patients with normal IGF-I levels had tumor recurrence. Ronchi et al. concluded that although minor abnormalities of GH suppression might persist in some patients, normalization of IGF-I levels generally indicated an excellent prognosis.

Other observational studies compared the frequency of normal IGF-I values to suppressed GH in patients with acromegaly. Nomikos et al. [3] studied 506 out of 688 patients who had been treated surgically. Cures occurred in $57.3 \%$ of patients, as defined by a basal GH less than $2.5 \mathrm{ng} / \mathrm{ml}$ (or a response to oral glucose less than $1 \mathrm{ng} / \mathrm{ml}$ ). Importantly, in this large series, the percentage of subjects who had a normal post OGTT GH value and an IGF-I in the normal range were similar. Therefore, the authors concluded there were not significant differences between measuring one parameter or the other in terms of defining cure.

Another study compared the response to conventional radiation therapy. Jenkins et al. [4] studied 1080 subjects, 856 of whom had received conventional RT. After 10 years, $60 \%$ of patients had a GH value less than $2 \mathrm{ng} / \mathrm{ml}$ and $63 \%$ had a normal IGF-I level. Although the number of discrepancies was not reported, it is significant that the same percentage had this degree of improvement in both parameters, suggesting that there was a high level of concordance between the two measurements.

A few studies have examined the degree of improvement in GH and IGF-I levels after surgery, studying the correlation of biochemical responses with improvement in signs or symptoms of disease activity. Clemmons et al. [5] demonstrated that IGF-I at diagnosis corresponded with soft tissue enlargement $(r=0.77)$, as well as with the degree of abnormality in fasting blood glucose and blood glucose after OGTT. In contrast, the glucose-suppressed GH had correlation coefficients that were much lower $(r=0.32-0.35)$. The authors concluded that IGF-I values were more directly related to changes in soft tissue enlargement and insulin resistance than GH levels after an OGTT.

Puder et al. [6] measured a symptom index of acromegaly consisting of sweating, arthralgias, perception of soft tissue thickening, and headaches in patients who had been stratified into 3 groups using biochemical cure criteria: group 1 (normal IGF-I and completely normal GH values after OGTT); group 2 (normal IGF-I but GH did not suppress to normal); and group 3 (abnormal IGF-I). The symptom index in group 1 (biochemical cure) was extremely low as was that in group 2 . In contrast, patients with elevated IGF-I (group 3) had markedly increased symptoms and reduced insulin sensitivity. The authors concluded that IGF-I was a better indicator of abnormal insulin sensitivity and the persistence of clinical symptoms.

Dimaraki et al. [7] also evaluated IGF-I and frequent GH sampling measurements as parameters for the diagnosis of acromegaly in 16 patients. Subject inclusion criteria were elevated plasma IGF-I levels and mean plasma GH $<5 \mathrm{ng} /$ $\mathrm{ml}$. The mean GH level was $<2.5 \mathrm{ng} / \mathrm{ml}$ among $25 \%$ of patients during frequent sampling. The OGTT-GH nadir was $<1 \mathrm{ng} / \mathrm{ml}$ in 8 out of 16 patients and in one patient was less than $0.14 \mathrm{ng} / \mathrm{ml}$ cutoff $(<0.21 \mathrm{ng} / \mathrm{ml}$ cutoff when corrected for assay standards), further demonstrating the limitations of using GH levels as a cutoff. Post transsphenoidal surgery normalization of IGF-I, occurring in 11 of 14 patients, was associated with improved morbidity despite normal preoperative GH. They concluded that IGF-I was the most sensitive and reliable test of active acromegaly.

Pivonello et al. [8] studied the relationship of cardiac structural changes with persistence of abnormal IGF-I or GH values in 17 patients treated with pegvisomant $10 \mathrm{mg} /$ day up to $40 \mathrm{mg} /$ day for 6 to18 months. Left ventricular ejection fraction (LVEF) was found to be the most sensitive predictor of worsening symptoms. Although the value of $\mathrm{GH}$ as a predictor could not be assessed because the GH receptor antagonist was used, functional improvement in LVEF correlated with improved serum IGF-I $\left(R^{2}=0.66\right)$. The high correlation between improvement in LV function and IGF-I suggests that this would be a good marker for prediction of disease control.

A study by Colao et al. [9] examined the relationship between change in tumor size and percent decrease in IGFI and GH among 99 patients treated with somatostatin analogs. The percent decrease in IGF-I was a better marker of tumor shrinkage (volume on magnetic resonance imaging [MRI]) as compared to the change in GH levels. The percent change in tumor volume correlated with the percent 
change in IGF-I, $\left(R^{2}=0.2, P<0.004\right)$. This strongly suggests that the percent decrease in IGF-I was the best predictor of tumor shrinkage.

Recently a large study by Alexopoulou et al. reported on all non-cured patients (229 patients) in the Belgium acromegaly registry [10]. Disease was classified as controlled versus active depending upon symptomatic index and other indices, such as continued acral growth or tumor enlargement. Concordance between IGF-I and GH was observed among $65 \%$ of the patients (35\% with both GH and IGF-I high, whereas the other $30 \%$ had controlled disease and normalization of both parameters). Discordant values were observed in the remaining $35 \%$ of the patients (24\% $(n=55)$ with persistent abnormally high IGF-I levels in the presence of controlled GH and $11 \%(n=25)$ with high GH levels in the presence of a normal IGF-I). Importantly, the authors felt that the subgroup with high GH and normal IGF-I levels were for the most part well controlled in that they had a normal OGTT and an absence of anthropometric or radiologic indication of disease progression. Several of these subjects were younger women who were still having menstrual cycles suggesting that gonadal steroids might be the cause of discordance between IGF-I and GH values. In contrast, patients with elevated IGF-I levels had a worse metabolic profile and other evidence of disease progression. The authors concluded that measurement of IGF-I was a more accurate index of active disease and a worse outcome.

Three studies reported QOL measurements and the relationship between them and both GH and IGF-I values during treatment. Paisley et al. [11] found a correlation between decreased IGF-I and overall improvement in QOL among 56 patients who were administered the AcroQol questionnaire (where the correlation coefficient between the change in QOL and change in IGF-I was $r=-0.36, P<0.006$ ). However, GH was not measured in these subjects.

Kauppinen et al. [12] measured health-related QOL among 231 subjects at follow-up, with a mean of 11.4 years post initial therapy, using a 15-dimension instrument (15D). The best QOL scores were achieved when GH was suppressed post OGTT to between $0.3 \mu \mathrm{g} / \mathrm{l}$ and $1 \mu \mathrm{g} / \mathrm{l}$ and also when IGF-I was normal. However, normal IGF-I neither predicted cure of diabetes mellitus or hypertension nor did it correlate with an improved QOL score. Basal GH at diagnosis and GH levels during treatment also did not correlate. Twenty-three percent had discordant GH and IGF-I values, but comparable improvements in QOL.

Trepp et al. [13] compared QOL among 33 patients with treated acromegaly. IGF-I was found to be a statistically significant predictor in improvement in health-related QOL per the total AcroQol score (mean of $-0.8 \%$ per $10 \mathrm{ng} / \mathrm{ml}$ increase in IGF-I, 95\% confidence interval (CI), -1.4-0.1, $P=0.018$ ). GH results were not included other than to report that $18 \%$ of subjects had discordant GH and IGF-I measurements.

Because acromegaly is associated with a 10-year shortening of lifespan, a test to predict those patients who are most likely to have premature mortality would be of great value. Seven studies have reported the value of GH or IGF-I levels in predicting premature mortality from acromegaly, however only one study formally measured GH and IGF-I in all subjects. This study reported by Holdaway et al. [14] demonstrated that both GH suppression and IGF-I normalization correlated with improved mortality rates. Subjects with a raised IGF-I greater than the $95 \%$ confidence interval had a 3.4-fold increased standardized mortality ratio (SMR) whereas those with IGF-I less than 2 standard deviations below the mean had only a 1.2-fold increase. Subjects with elevated GH showed a 2.6-fold increase in SMR if GH was not suppressible to $<5 \mu \mathrm{g} / \mathrm{l}$. However, if GH was suppressible to less than $2 \mu \mathrm{g} / \mathrm{l}$, the SMR was statistically significantly increased, but only 1.6- fold. Therefore, the degree of risk was greater with an elevated IGF-I compared with an elevated GH.

In another publication by Holdaway et al., the authors performed a meta analysis on all studies that measured the efficacy of GH or IGF-I to predict mortality during followup treatment [15]. This study showed that the SMR was significantly elevated for increased IGF-I in 6 of 7 reported studies, however $95 \%$ confidence interval crossed the line of unity in 3 of 6 . A normal IGF-I also predicted no increase in mortality in 7 of 7 reported studies. In contrast, GH values were reported to show an increased SMR in 8 of 10 studies and 4 of 10 crossed the line of unity.

It should be noted that use of somatostatin analogs may complicate the interpretation of GH suppression tests and result in discordance between GH and IGF-I levels. This may be because GH does not always suppress in response to glucose in the presence of somatostatin analogs, or that treatment with somatostatin analogs may exert a direct effect on liver production of IGF-I. Therefore, caution has to be used in interpreting some IGF-I assays. Because IGF-I varies with age and BMI, a large number of normative subjects have to be used in order to obtain reliable normal reference ranges [16].

Grading of evidence

Approximately half of the Summit panelists (56\%) concluded the evidence available was Category II, 33\% considered it be Category III, and $11 \%$, Category I (Table 2).

Level of support

All Summit participants voted to accept the statement although $78 \%$ had some reservations. Survey results 
Table 2 Summit panel voting and grading schemes

\begin{tabular}{ll}
\hline Category & Nature of evidence \\
\hline I & Evidence obtained from at least 1 well-designed, randomized, controlled trial \\
II & Evidence obtained from well-designed cohort or case-control studies \\
III & Evidence obtained from case series, case reports, or flawed clinical trials \\
IV & Opinions of respected authorities based on clinical experience, descriptive \\
& studies, or reports of expert committees \\
V & Insufficient evidence to form an opinion \\
& Individual level of support (Panel members) \\
1 & Accept recommendation completely \\
2 & Accept recommendation with some reservations \\
3 & Accept recommendation with major reservations \\
4 & Reject recommendation with reservations \\
5 & Reject recommendation completely \\
\hline
\end{tabular}

revealed that community physicians in endocrinology and neurosurgery felt similarly: $84 \%$ voted to accept the statement completely or with some reservations; $10 \%$ with major reservations; and the remaining $6 \%$ rejected the statement with reservations (Fig. 2).

\section{Discussion}

As suggested by Summit panel voting and survey participants, the value of IGF-I in monitoring for effective control of acromegaly is supported by a growing body of evidence. Panelists suggest that reservations regarding the statement's validity stem more from the lack of normative data required for optimizing usefulness of IGF-I assays in clinical practice than from the statement itself.

\section{Future direction}

In summary, several studies have demonstrated the efficacy of both GH and IGF-I measurements in monitoring acromegaly. The evidence currently suggests that elevated IGF-I is a better predictor of persistent symptoms and signs, indicating a need for further therapy.

Both measurements predict an increase in SMR and therefore, both will yield at least equivalent information with regard to long-term increased mortality risk. For these reasons, it is recommended that both measurements be performed in post-treatment acromegalic subjects at least once. If the results are discordant, IGF-I should be followed as an indicator for a need for treatment of persistent symptoms, and either IGF-I or GH can be followed as an indicator for need for treatment reducing risk of premature mortality.

Because of the importance of IGF-I assays in the treatment of acromegaly, individual IGF-I assays must provide more uniform scientifically validated standards and robust normative data. In addition, although gender effects clearly influence IGF-I levels, available assays do not provide normative data based on gender or use of gonadal steroid hormones.

\section{Statement 2: a $\mathbf{G H}$ of $\leq \mathbf{1 . 0} \mu \mathrm{g} / \mathrm{l}$ as a random measurement or as a nadir after an oral glucose tolerance test correlates with disease control in patients with acromegaly}

Rationale and definition of statement

Patients with acromegaly have increased mortality and morbidity. Multiple modalities are now available for the treatment of acromegaly, including surgery, irradiation (conventional and stereotactic), and medical. The ultimate goal of treatment is resolution of symptoms, control of tumor mass and normalization of mortality. However, because most tumors are macroadenomas and often invasive, complete surgical cure is rarely achieved.

It is important to determine the levels of GH and/or IGF-I that are associated with improvement of the increased mortality and morbidity. This assesses the strength of evidence supporting the assertion that a $\mathrm{GH}$ of $\leq 1.0 \mu \mathrm{g} / \mathrm{l}$ as a random measurement or as a nadir after an OGTT correlates with disease control in patients with acromegaly and, therefore, should be a goal of therapy.

\section{Literature search}

Because the evidence of disease control is by definition the normalization of mortality and resolution of symptoms, a PubMed database search to identify studies related to morbidity and mortality in patients with acromegaly was completed on October 2008. The search terms used were "acromegaly," "mortality," and "morbidity." Reference lists of pertinent articles were also reviewed to see if studies missed by the PubMed search were appropriate for inclusion. Studies that assessed the morbidity and mortality 
of cohorts of acromegalic patients longitudinally and correlated these outcomes with GH and IGF-I levels were selected. None of these were controlled studies in which some patients are not treated. Most compared morbidity and mortality in acromegalic patients to age-adjusted and sex-adjusted controls living in the same area/country and then stratified patients by GH and IGF-I levels.

\section{Evidence}

\section{Increased mortality in acromegaly}

Although many studies have reported increased mortality in patients with acromegaly, there have not been any in which patients with acromegaly were randomized to treatment versus no treatment and then followed over time. In all series, patients were treated by a variety of modalities and then mortality rates were computed as SMRs compared to a matched population without acromegaly. As early as 1980, Alexander et al. reported an SMR of 3.3 in 164 patients with acromegaly, with the increased deaths being attributable to cerebrovascular, cardiovascular, respiratory, and oncologic causes [17]. Holdaway reviewed the literature in 2007, finding 18 series in which the SMR was reported [18]. In this review, the SMR ranged from the 3.3 reported by Alexander et al. [17] to 1.16 reported by KauppinenMakelin et al. [19], with the average for the series being approximately 2.0 [18]. Increased mortality rates in patients who had received radiotherapy as part of their treatment were reported frequently [18]. Some studies also showed increased mortality rates in subjects with diabetes and hypertension and in those with delays between diagnosis and treatment [14]. Overall, there appeared to be improvement in outcomes over time, with lower mortality rates in more recent studies compared to older ones [18]. This is likely due to better methods of treatment, improved treatment of comorbidities, the use of somatostatin analogs, and increasingly stringent hormone goals for treatment [18].

Growth hormone goal of $<2-2.5 \mu \mathrm{g} / \mathrm{l}$

Control of GH has been a treatment goal for almost 20 years. However, there has been no consensus in how the GH should be measured, hampering the ability to compare data from different studies. Methods include obtaining samples over the course of several hours and then averaging the results ("day curve"). Alternatively, an OGTT is performed and only the nadir GH is reported. In other studies, a "random" GH measurement is obtained, although this was often done fasting in the morning. Further complicating this issue are changes in GH assays over the past two decades, with improvement in sensitivity as they changed from the standard radioimmunoassay (RIA) employing polyclonal antibodies to current chemiluminescence methods employing monoclonal antibodies [20]. Although centers in most countries have reported GH values in mass units of $\mu \mathrm{g} / \mathrm{l}$, centers in the United Kingdom usually reported GH values as $\mathrm{mU} / \mathrm{l}$. Conversion values to mass units were not always included in reports and not all assays were equivalent in these conversion values. In most of the discussion to follow, random GH measurements were performed using older RIAs.

In the early 1990s, the goal for treatment was GH level $<5.0 \mu \mathrm{g} / \mathrm{l}$. Bates et al. reported that in their long-term follow-up study of 79 patients, those with GH levels $>5 \mathrm{mU} / \mathrm{l}$ using a 5-point day curve had an SMR of 3.32, whereas those with GH levels $<5 \mathrm{mU} / \mathrm{l}$ had an SMR of 1.42 [21]. Unfortunately, they did not report the conversion value for their assay in terms of mass units [21]. In a study of 254 patients who had undergone transsphenoidal surgery, Abosch et al. found that the SMR for individuals with a $\mathrm{GH}>5.0 \mu \mathrm{g} / \mathrm{l}$ was 3.10 whereas it was 1.00 for those with $\mathrm{GH}<5.0 \mu \mathrm{g} / 1$ [22].

More recent studies have addressed benefits of lowering GH levels to less than $2.0-2.5 \mu \mathrm{g} / \mathrm{l}$. In a retrospective analysis of 1362 patients from 15 centers in the United Kingdom, Orme et al. found SMRs of 1.10 (CI, 0.89-1.35), 1.41 (CI, 1.16-1.68), and 2.12 (CI, 1.70-2.62), in patients with GH levels of $<2.5,2.5-9.9$, and $\geq 10 \mu \mathrm{g} / 1$, respectively [23]. When they looked at the causes of mortality, a linear trend for increasing mortality with increasing GH levels was found for cardiovascular and malignant disease, but not for respiratory or cerebrovascular disease [23]. In a Canadian study of 103 patients, Beauregard et al. found that patients in remission, defined as a random $\mathrm{GH}<2.5 \mu \mathrm{g} / \mathrm{l}$ or a glucose suppressed GH $<1 \mu \mathrm{g} / \mathrm{l}$ and a normal IGF-I, had an SMR of 0.88 , whereas those not in remission had an SMR of 4.8 [24]. Similarly, Kauppinen-Makelin et al. found that of 334 Finnish patients, those with a random $\mathrm{GH}<2.5 \mu \mathrm{g} / \mathrm{l}$ had an SMR of 0.48 (CI, 0.23-0.88) whereas those with levels $\geq 2.5 \mu \mathrm{g} / 1$ had an SMR of 1.63 (CI, 1.10-2.35) [19]. Mestrón et al. reported a retrospective analysis of 1219 Spanish patients that used GH (basal or OGTT-derived nadir $<2 \mathrm{ng} / \mathrm{ml}$ ) with or without concomitant normalized IGF-I levels or normalized IGF-I alone as the criteria for a cure. Significantly more people who had never achieved a basal GH $<2 \mu \mathrm{g} / \mathrm{l}$ died (7.2\% of uncured patients) compared with those who did obtain a basal $\mathrm{GH}<2 \mu \mathrm{g} / \mathrm{l}$ (1.3\% of cured patients), $P<0.001$ ). Similar numbers were reported when the cure was defined using OGTT-derived GH nadir $<2 \mu \mathrm{g} / \mathrm{l}$ as the cutoff [25]. Unfortunately, they did not calculate SMRs in their study [32]. On the other hand, in 3 smaller studies of Swiss $(n=94)$ [26], Japanese $(n=154)$ [27], and Dutch $(n=164)$ [28] acromegalic patients, only small nonsignificant differences in SMR with GH levels above and below the $2.5 \mu \mathrm{g} / \mathrm{l}$ cutoff were found. 
Only two studies attempted to determine whether GH levels $<1 \mu \mathrm{g} / \mathrm{l}$ resulted in a further decrease in mortality. In their study of 208 acromegalic patients from New Zealand, Holdaway et al. found that the SMRs for GH levels $<1,<2$ and $<5 \mu \mathrm{g} / 1$ were 1.1 (CI, 0.5-2.1), 1.6 (CI, 0.9-3.0), and 2.5 (CI, 1.6-3.6) [14]. The value for $<2 \mu \mathrm{g} / \mathrm{l}$ was not significantly increased and not statistically different from that for $<1 \mu \mathrm{g} / \mathrm{l}$. Ayuk et al. analyzed their database of 419 acromegalic patients from the West Midlands region of the United Kingdom, finding a clear but only borderline statistically significant $(P=0.068)$ trend mortality benefit with a GH cutoff of $2 \mu \mathrm{g} / \mathrm{l}$ [29]. Furthermore, they found not even a trend towards further benefit using a GH cutoff of $1 \mu \mathrm{g} / 1$ [29].

Recently, Holdaway et al. performed a formal metaanalysis of the effect of lowering $\mathrm{GH}$ levels to $<2.5 \mu \mathrm{g} / \mathrm{l}$, abstracting data from the studies mentioned above. In this analysis of 10 studies, they found a pooled SMR of 1.9 (CI, 1.5-2.4) for patients with final $\mathrm{GH}$ levels $>2.5 \mu \mathrm{g} / \mathrm{l}$, whereas the pooled SMR was 1.1 (CI, 0.9-1.4) for those with final GH levels $<2.5 \mu \mathrm{g} / \mathrm{l}$ (Fig. 1) [15].

Interestingly, in this meta-analysis, there was a clear improvement in the overall SMR over time. The SMR for the 6 studies in which the mean year of patient entry was prior to 1984 was 2.2 (CI, 1.8-2.8) and that for the 7 studies in which the mean year of patient entry was after 1984 was 1.3 (CI, 1.1-1.6) [15].

Grading of evidence

Based on a review of the 11 studies presented above as well as the meta-analysis performed by Holdaway et al. [15], the 9 members of this workshop varied widely in opinion regarding the nature of the evidence to support/refute this statement. Grades 2 and 5 were chosen by $22 \%$ each whereas $44 \%$ chose grade 3 and $11 \%$ chose grade 4 . See Table 2.

Level of support

Seventy-eight percent of the Summit participants voted to accept the statement, primarily with major reservations, compared with $94 \%$ of the respondents surveyed, who accepted it either completely or with some reservations. It was rejected by 22 and $6 \%$ of the Summit participants and survey participants, respectively. More detailed results can be found in Fig. 2.

Discussion

Major reservations regarding the acceptance of the statement became evident. Panelists identified several shortcomings of
Fig. 1 Pooled standardized mortality ratios (SMRs) in studies of acromegaly grouped by GH level at final follow-up [15]. (C) European Society of Endocrinology (2008). Reproduced by permission. Note: Data are SMR $(95 \%$ confidence interval)

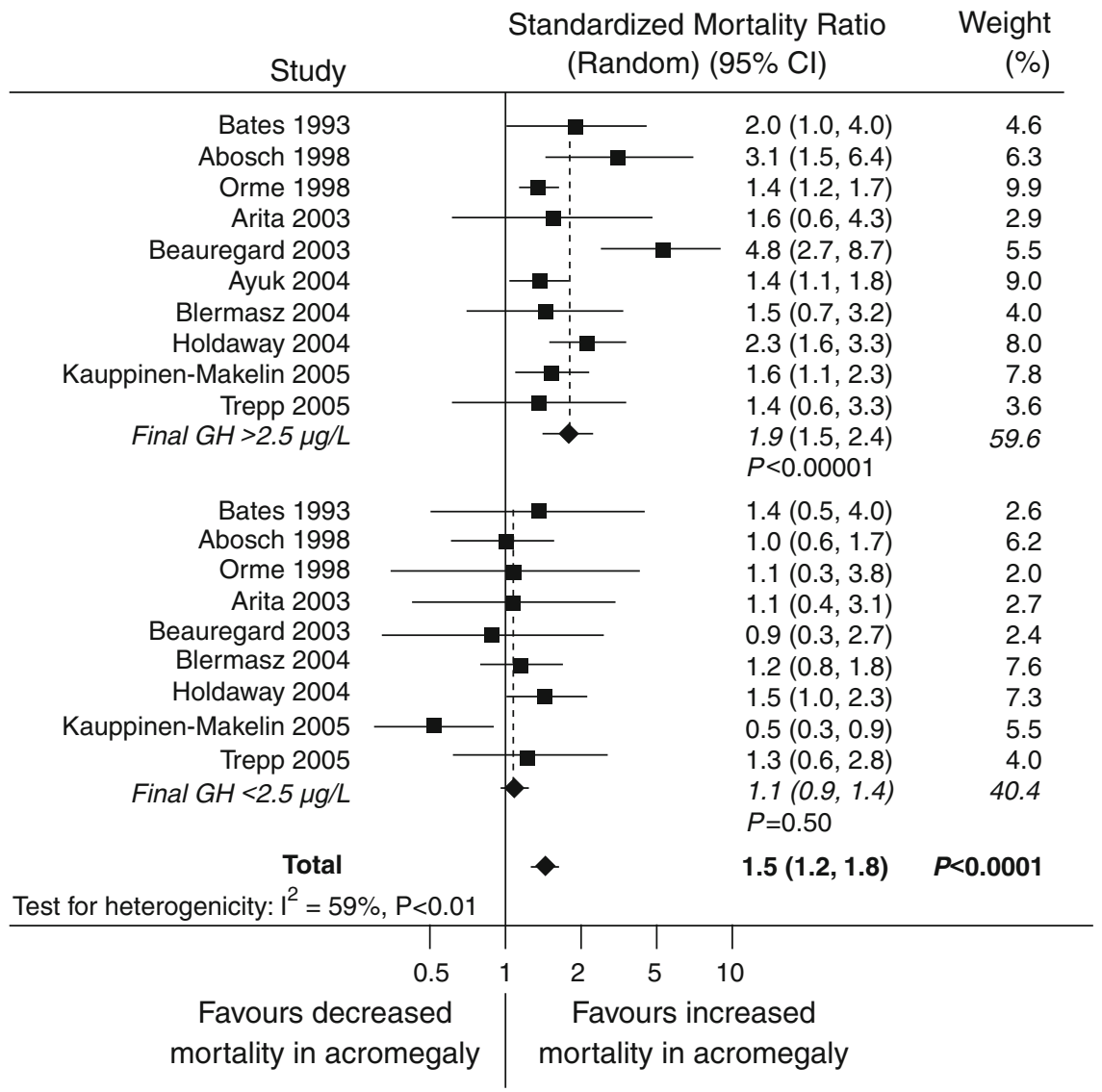




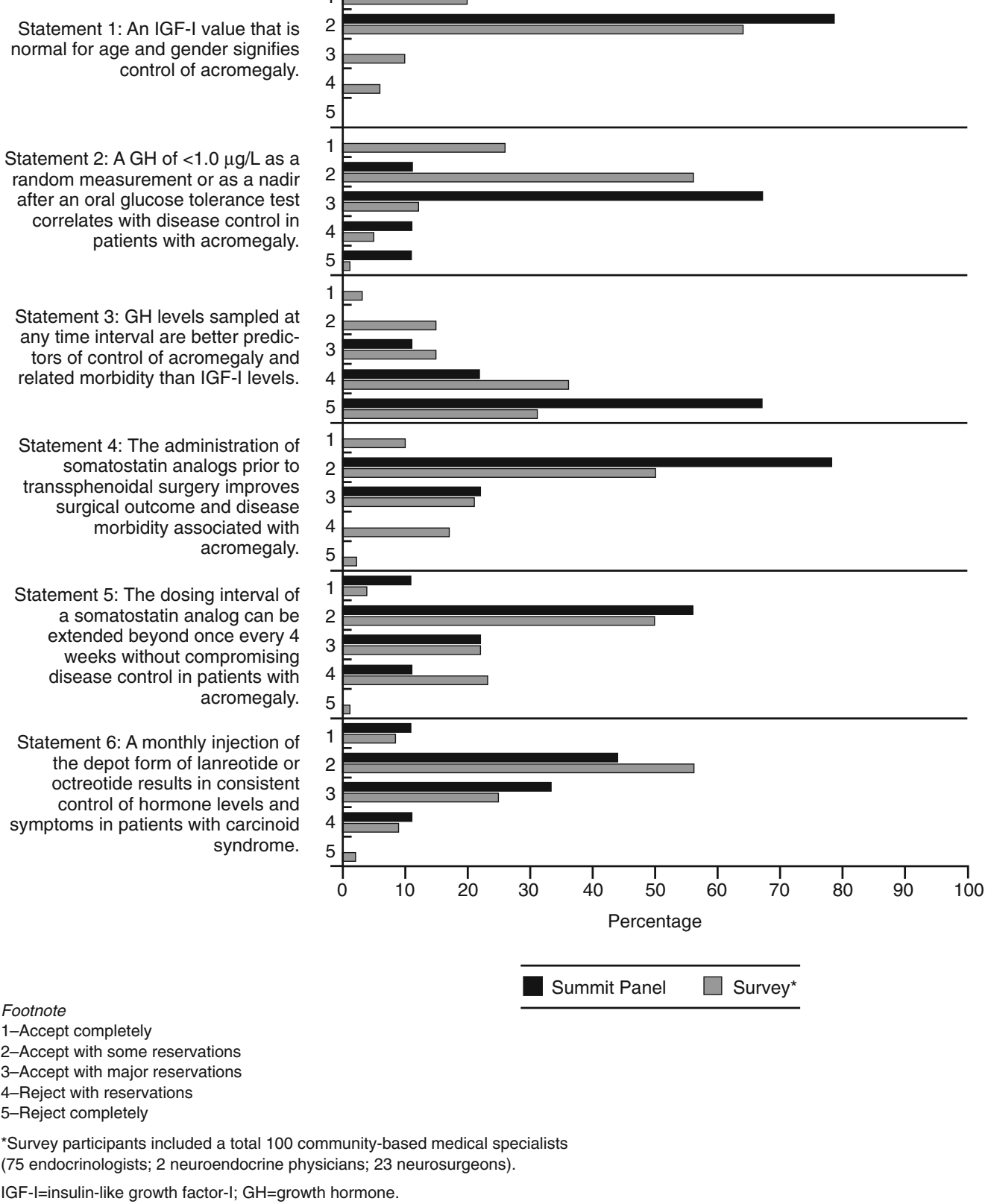

Fig. 2 Comparison voting between summit panel and surveyed clinicians for 6 statements related to the current treatment of acromegaly and carcinoid syndrome

available data, which included variability in current GH assays as well as changes in $\mathrm{GH}$ assays and treatments over time. Others included lack of control or data on comorbidities and cause of death. There were very little data available examining the use of OGTT-derived GH values instead of random or basal GH values. Therefore, this aspect of the statement was not well supported.

As mentioned earlier, the presence of disease control was measured by the mortality rate, despite the possibility that symptom control or QOL might also be considered a component of disease control. However, all of the longterm outcome data primarily focuses on mortality rather than morbidity. The few data available suggest that morbidity tracks very closely with mortality with respect to $\mathrm{GH}$ levels $[30,31]$ and that there is no need to use different criteria for morbidity compared to mortality.

Data reviewed supports a cutoff of $2.5 \mu \mathrm{g} / \mathrm{l}$ using older assays. Furthermore, Summit participants recognized that 
the statement's cut-off $\mathrm{GH}$ level of $1 \mu \mathrm{g} / \mathrm{l}$ using current assays approximates the GH levels of about 2-2.5 $\mu \mathrm{g} / 1$ using older RIAs in the studies reviewed. Thus, they recommended that a current goal of therapy for patients with acromegaly should be $\mathrm{GH}<1 \mu \mathrm{g} / 1$ since a $<2.5 \mu \mathrm{g} / \mathrm{l}$ (as assessed by older assays in past studies) correlated with normalization of the high mortality rate associated with acromegaly.

\section{Future direction}

Panelists stressed the importance of the differences between the older and newer GH assays when trying to formulate goals for treatment. Not only are the assays of different sensitivities, but they also vary in how they correlate with $\mathrm{GH}$ standard preparations. It will be important in future studies to specify the type of GH assay used and how it relates to appropriate $\mathrm{GH}$ standards. There was no uniformity among the Summit participants as to whether a single random $\mathrm{GH}$ specimen versus $\mathrm{GH}$ after oral glucose (or GH day curves) should be obtained. However, from a practical point of view, it was recognized that a single specimen is the most practical way to follow patients over time, and that a fasting specimen is preferred to a "random" specimen. It was also recognized that in earlier studies, determination of cause of death was often declared according to local custom and regulations. For future studies, standardized methods should also be used in determining mortality and causes of death as well as morbidities. Every effort should also be made to aggressively manage the comorbidities of acromegaly since those conditions have the potential to impact outcomes as does the management of GH and IGF-I levels.

\section{Statement 3: GH levels sampled at any time interval are better predictors of control of acromegaly and related morbidity than IGF-I levels}

\section{Rationale and definition of statement}

Determination of the best methods for monitoring treatment effectiveness is essential for optimal care of patients with acromegaly. While control of the disease and related morbidity are the true treatment goals, methods of predicting and measuring the adequacy of treatment depend on the use of biochemical measurements such as serum GH and IGF-I levels as interim markers of success or failure. Whether one marker extends advantages over the other, perhaps even to the extent of eliminating the need for a second marker, is the topic being considered here. How to interpret discrepant results between these parameters is also discussed here since such discrepancy is not rare.
Literature search

A literature search in PubMed was performed in October 2008, revealing 94 articles relating to GH, IGF-I, acromegaly, morbidity, and mortality. Of these, 11 were considered to be most pertinent to the statement.

\section{Evidence}

A recent review article reported a meta-analysis of mortality studies, which included data from 4806 patients and 1116 deaths [15]. It demonstrated that mortality rates in patients with random serum $\mathrm{GH}<2.5 \mu \mathrm{g} / \mathrm{l}$ (measured primarily with RIA) despite treatment were close to expected (SMR 1.1 [95\% CI, 0.9-1.4]) in contrast to patients with $\mathrm{GH}>2.5 \mu \mathrm{g} / \mathrm{l}$ (SMR of 1.9 [95\% CI, 1.5-2.4]). The authors concluded that the random serum GH cutoff should be lowered to $1 \mu \mathrm{g} / \mathrm{l}$ if modern sensitive immunoassays are used. Findings were similar when IGF-I was the criteria, with an SMR of 1.1 (95\% CI, 0.9-1.4) in patients with serum IGF-I normal for age and sex at last follow-up after treatment compared with 2.5 (95\% CI, 1.6-4.0) in those with elevated IGF-I levels.

In the Belgian registry enrolling 229 non-controlled patients [10], 35\% had a discordant GH and IGF-I pattern. Young estrogen-sufficient females tended to make up the group of the high $\mathrm{GH}$ phenotype, suggesting that age, gender, and estrogens have a role in this divergence. On the other hand, the high IGF-I phenotype was associated with a worse metabolic profile suggesting that it might indicate persistent active disease.

Another study presented data from 166 patients who underwent multiple GH and IGF-I testing as well as an OGTT following either surgery or during treatment with somatostatin analogs or dopamine agonists [32]. Discordant results of OGTT testing were noted in 32,48 , and $18 \%$ in the 3 treatment groups, respectively. In patients studied during somatostatin analog therapy, $42 \%$ of tests were discordant with a pattern of normal IGF-I and GH nadir $>1 \mu \mathrm{g} / 1$, while only $4 \%$ of tests had similar discrepancy in patients treated with dopamine agonists. The discordance between GH and IGF-I control in this treatment group were similar when fasting GH levels were used, leading to the conclusion that both basal and $\mathrm{GH}$ nadir levels are highly discordant with IGF-I levels during somatostatin analog therapy (significantly different compared with surgery group $[P=0.03]$ and dopamine agonist group $[P<0.001]$ ) and that OGTT is not useful in assessing biochemical control in these subjects [32].

There are several studies that investigate predictors of discrepancy between GH and IGF-I. As already mentioned, estrogen-sufficient women or those receiving estrogen therapy for hypogonadism are more likely to have elevated $\mathrm{GH}$ levels, both in a fasting state [10, 33, 34] and after 
glucose load [1] in presence of normal IGF-I levels. Indeed, withdrawal of oral estrogen replacement during chronic treatment with somatostatin analogs was associated with significantly lower GH levels and higher IGF-I levels than during treatment [33]. A gender difference in the relationship between serum GH and IGF-I in 153 patients with active acromegaly was confirmed in another study, where results were again consistent with relative $\mathrm{GH}$ resistance in normal and GH-deficient females; these findings were considered partially mediated by estrogens. It was estimated that serum IGF-I values for a given serum $\mathrm{GH}$ value were $82 \mathrm{ng} / \mathrm{ml}$ less in females than in males $(P<0.02 ; 95 \% \mathrm{CI}$, 15.2-149). The mean serum IGF-I for a given GH value was $130 \mathrm{ng} / \mathrm{ml}$ lower in females on oral estrogen therapy than in males $(P=0.01 ; 95 \% \mathrm{CI}, 29.8-230.2)$, but were only $60 \mathrm{ng} / \mathrm{ml}$ less than the other 45 females $(P=0.2)$ [35].

Similarly, older patients, as well as those with a higher BMI, generally had lower GH levels, either as fasting or post-glucose values $[1,36]$. In patients aged above 60 years, cutoff $\mathrm{GH}$ levels as low as $1.4 \mu \mathrm{g} / \mathrm{l}$ (as a fasting sample) and $0.5 \mu \mathrm{g} / \mathrm{l}$ (as a post-glucose nadir level) were proposed [36]. However, another study found $\mathrm{GH}$ levels (either basal or post-glucose nadir) to be no different based on age or gender [37]. It is evident that normative GH data still needs to be fully established.

One potential source of bias in analyzing $\mathrm{GH}$ values during treatment of acromegaly is the assay used. A recent study investigated the measurement of GH during a standard 75-g OGTT in 46 acromegaly patients and 213 healthy subjects. This study used 3 different commercially available assays (Immulite [Diagnostic Products Corp., Los Angeles, CA]; Nichols [Nichols Institute Diagnostika GmbH, Bad Vilbel, Germany]; and Diagnostic Systems Laboratories [Sinsheim, Germany]) that were calibrated against recently recommended GH standards [38]. Although $\mathrm{GH}$ results from all 3 assays strongly correlated with each other, those obtained with the Immulite assay were 2.3- and 6-fold higher than those obtained with Nichols or Diagnostic Systems Laboratories, respectively. Different cutoff limits ( $1 \mu \mathrm{g} / \mathrm{l}$ [Immulite] and $0.5 \mu \mathrm{g} / \mathrm{l}$ [Nichols]) were found to identify $95 \%$ of patients with active disease and $78-80 \%$ of patients in remission [38]. The authors confirmed that significantly higher basal and nadir GH levels occurred in healthy females than in males (Immulite $2.2 \pm 0.28$ vs. $0.73 \pm 0.15 \mu \mathrm{g} / \mathrm{l}$ for basal results and $0.16 \pm 0.01 \mathrm{vs}$. $0.08 \pm 0.01 \mu \mathrm{g} / \mathrm{l}$ for nadir results; $P<0.001$, respectively). Gender-specific differences were also noted in acromegalic patients, although not statistically significant. Multiple regression analysis revealed age, BMI, and gender to be predictors for basal and nadir GH levels, and that post-glucose GH-nadir values (and likely fasting $\mathrm{GH}$ values) are assay-, gender-, age-, and BMI-specific [38]. These data indicate that the GH cutoff currently used presents important drawbacks, explaining the high rate of discrepancy with IGF-I levels. They also suggest that GH cutoffs should be age-, gender-, and BMI-related as are IGF-I ranges. Moreover, the data suggested a need for cutoff limits to be assayindividualized.

These problems in accurately assessing the biochemical control of acromegaly might explain why some data report similar clinical improvement in patients both who are well controlled biochemically and those who are not. Mean daily GH levels, IGF-I, leptin and lipid levels, glucose, insulin, and GH nadir during OGTT were measured in 41 patients post transsphenoidal surgery for a $\mathrm{GH}$-secreting pituitary adenoma and in 23 patients with naive acromegaly serving as positive controls [39]. Additionally, insulin resistance was measured by homeostatic model index (R-HOMA), body composition assessed by dual-energy X-ray absorptiometry, and left ventricular mass index $\left(\mathrm{LVM}_{\mathrm{i}}\right)$ and cardiac index $\left(C_{i}\right)$ determined by echocardiography [39]. The authors reported no difference in cardiac indices, insulin resistance, body composition, or leptin levels between patients with complete biochemical remission and those with inadequately controlled disease after surgery. Cardiac index was lower in cured patients compared with naive patients and $\mathrm{LVM}_{\mathrm{i}}$ was similarly decreased in controlled (108.4 $\left.\pm 30 \mathrm{~g} / \mathrm{m}^{2} ; P=0.015\right)$ and inadequately controlled disease $\left(108.8 \pm 30.7 \mathrm{~g} / \mathrm{m}^{2} ; P=0.03\right)$ in comparison with naive disease $\left(160.3 \pm 80.6 \mathrm{~g} / \mathrm{m}^{2}\right)$. In both controlled and uncontrolled disease, R-HOMA index was lower than in naive disease $(2.2 \pm 1.4 ; P=0.001$ and $3.1 \pm 2.0$; $P=0.05$ vs. $5.1 \pm 3.1)$ and leptin concentration was higher $(14.9 \pm 8.7 \mu \mathrm{g} / \mathrm{l} ; P=0.004$ and $12.8 \pm 7.8 \mu \mathrm{g} / \mathrm{l}$; $P=0.05$ vs. $7.4 \pm 3.8 \mu \mathrm{g} / \mathrm{l})[39]$. Cardiac $(P=0.04)$ and R-HOMA index $(P=0.009)$ were independent predictors of biochemical remission when considered solely on normal IGF-I. Insulin resistance $(P=0.02)$ and leptin level $(P=0.002)$ were independent predictors of normalized mean GH values. Accordingly, cardiac indices, insulin resistance, and body composition were not different in those patients with complete biochemical remission compared to those with discordant GH and IGF-I levels [39].

Similarly, in a study where strictly controlled acromegaly improving cardiac dysfunction demonstrated that systolic function at rest was decreased by $18 \%(P<0.01), \mathrm{LVM}_{\mathrm{i}}$ increased by $40 \%(P<0.04)$ and isovolumetric relaxation time increased by $19 \%(P<0.01)$ in patients with active acromegaly compared with those with inactive disease. No difference was observed between patients cured after surgery or controlled with somatostatin analogs [40]. The ratio between early and late diastolic velocity (E/A ratio) using tissue Doppler imaging was decreased in active acromegaly compared with inactive $(0.75 \pm 0.07$ vs. $1.24 \pm 0.15$; $P<0.01)$, but was higher in surgically cured than in octreotide-treated patients $(1.75 \pm 0.41$ vs. $1.05 \pm 0.1$; 
$P<0.01)$. The fact that acromegalic patients who had been under long-term control with octreotide were likely to have diastolic function persistently and significantly more impaired than surgically cured patients suggests the existence of underlying biological effects of subtle abnormalities in GH secretion. However, a more recent prospective study [41] did not support the conclusion of the van Thiel study [40]. Further data on cardiac function are needed.

\section{Grading of evidence}

The Summit participants varied in their view of the nature of evidence as it pertains to the statement. Almost half (44\%) considered it to be of Category III whereas $22 \%$ thought it to consist of Category I and Category II each. One person considered it to be insufficient to form an opinion. See Table 2.

\section{Level of support}

Eighty-nine percent of the Summit participants voted to reject the statement compared with $67 \%$ of survey participants. Summit participants were more likely to reject it without reservations (Fig. 2).

\section{Discussion}

Serum GH measurements taken either at random times or as part of dynamic testing using the oral glucose suppression test were found to be indicative of disease control. The requirement of using both biochemical parameters to evaluate the activity of acromegaly is still valid. However, as the evidence demonstrates, there are too many uncertain aspects of GH testing to allow it a more dominant role in monitoring treatment of acromegaly.

\section{Future direction}

It would be helpful to reanalyze GH cutoff values according to patient's gender, age, BMI, gonadal status, and assay in previous studies, and to perform such analyses in future studies.

\section{Statement 4: the administration of somatostatin analogs prior to transsphenoidal surgery improves surgical outcome and disease morbidity associated with acromegaly}

Rationale and definition of statement

The use of somatostatin analogs has been demonstrated to be of benefit to patients with acromegaly. However, the role of surgical pretreatment in patients destined for transsphenoidal resection has yet to be defined. Consideration of the influence of somatostatin analogs on surgical outcomes, as well as surrogate results that may reflect on outcomes, are addressed here within the context of available clinical evidence.

\section{Literature search}

A literature search via the PubMed database was done in October 2008. The search terms "octreotide" and "lanreotide" using the "OR" function, yielded 6606 articles; "somatostatin analogs" yielded 4594; and "octreotide," "lanreotide," and "somatostatin analogs" combined by using the "OR" function yielded 9282 articles. The search terms "primary therapy" and "preoperative therapy" each yielded 298,217 and 113,760 articles, respectively; when these terms were combined using the "OR" function, 400,261 articles were found. The single term "acromegaly" yielded 7447 articles. And, 3 searches of all the listed terms combined with "AND" resulted in 138 articles. Upon examination, the search revealed 20 articles relevant to the statement, including 2 articles found in a hand review.

\section{Evidence}

The first studies considered here investigated the basic question of whether primary therapy with somatostatin analogs provides effective biochemical control of acromegaly. Results of these studies were included here as they serve as surrogate data for whether or not presurgical medical therapy is of benefit postoperatively.

In a published review of 24 studies that had been performed through 2005, Melmed et al. summarized the effects of primary treatment with somatostatin analogs among 424 patients. Thirty-seven percent of patients exhibited significant shrinkage, with an average of 50\% reduction in tumor size. One of the studies included in the review was a prospective open label study by Bevan et al. demonstrating that tumor shrinkage was associated with primary octreotide therapy in treatment-naïve patients [42, 43].

Baldelli's retrospective study among 118 patients presented compelling data showing non-inferiority of surgery versus radiotherapy versus primary therapy with lanreotide over a 24-month period. The ultimate levels of GH and IGF-I were similar in each group [44].

Three prospective open label studies subsequent to the 2005 critical analysis provided further surrogate data demonstrating that primary therapy with somatostatin analogs was associated with tumor shrinkage, and was effective for biochemical control. Colao et al. showed reduced tumor volumes in patients with microadenomas as 
well as macroadenomas [45]. In Cozzi's report of 67 patients, there was $62 \%$ mean tumor volume reduction. In this group, adenoma mass reduction to an empty sella was experienced by 11 patients, and cavernous sinus tumor invasion was resolved in some patients. Tumor shrinkage was noted to be more marked in younger patients. Most interestingly, one-third of patients who did not exhibit GH control still experienced tumor shrinkage. This was the first report of such discordance between tumor shrinkage and GH control in the literature [46]. Finally, Mercado et al. concluded that primary treatment with octreotide LAR was as effective as primary surgical resection [47].

Nine studies were identified which directly addressed the question at hand-does preoperative treatment enhance subsequent surgical outcome? The first 5 of these studies presented here concluded that surgical outcomes are not enhanced by prior use of somatostatin analogs. Three of these were prospective but nonrandomized studies by Biermasz, Kristof, and Plockinger which showed no differences in postoperative outcomes in patients pretreated with short-acting subcutaneous octreotide, or not [48-50]. Plockinger's study also showed no differences in shortterm or long-term GH control or in pituitary function parameters in the 2 groups [50].

Losa et al. reported a retrospective case-matched study with 286 patients that showed no difference in surgical outcomes. Patients were pretreated with any of different preparations of both octreotide and lanreotide for varying lengths of time [51]. Abe and Ludecke also showed data from reported results of a retrospective study among 147 patients who had received subcutaneous octreotide for at least 3 months preoperatively. The remission rates were lower in the medically pretreated group (69\%) when compared with the surgery-only group (77\%), although the differences did not achieve statistical significance [52].

The first of 4 studies that demonstrated improved surgical outcomes following prior treatment with somatostatin analogs is that of Barkan's, where 10 patients with untreated invasive pituitary macroadenomas were treated prospectively with subcutaneous octreotide for 3 to 30 weeks. Remission rates were significantly enhanced compared with those achieved in historical controls where patients were operated on by the same surgeon [53]. Stevenaert et al. performed a prospective open label study among 48 patients where preoperative octreotide was compared with surgery only. Remission rates were significantly higher in the group receiving octreotide pretreatment compared with surgery only, especially among patients with enclosed adenomas (89 versus 69\%; $P<0.05$ ), but outcome differences were not observed in the group of patients with invasive adenomas [54].

Colao et al. performed a retrospective study using a control group from the same 5-year time period which utilized the same surgical team. Patients in the pretreatment group received octreotide for 3 to 6 months prior to surgery. Clinical and surgical outcomes were both enhanced in the pretreated group. Postoperative circulating GH and IGF-I levels were controlled in 11 untreated $(29.7 \%)$ versus 12 octreotide-treated $(54.5 \%)$ patients $(P=0.005)$, respectively. Preoperatively, considerable tumor shrinkage was observed in 5 of the 22 patients and electrocardiogram (ECG) abnormalities normalized in $67 \%$ of patients pretreated with octreotide [55].

Carlsen's report details the only study that directly addressed the statement. This prospective, randomized, controlled study compared outcomes in patients who had received a 6-month or longer course of octreotide LAR preoperatively with those who underwent surgery without prior medical treatment. All patients were treated within a closed healthcare system that provided consistent methodologies for MRIs and laboratory assays, and utilized the same surgical teams. A substantially greater number of pretreated patients experienced biochemical remission compared with surgery alone (50 vs. $16 \%, P=0.017$ ). Despite the study randomization, the pretreatment group was found to exhibit significantly lower baseline serum IGF-I levels as compared with the surgery only group [56]. The effects of this confounding factor on the findings are not known.

Finally, the question can be asked-is there or could there be a potential difference between octreotide and lanreotide in terms of effects on surgical outcomes? Based on several studies directly comparing effects of octreotide LAR and lanreotide depot as reviewed by Murray et al., no difference was found between the 2 molecules in regard to GH and/or IGF-I responsiveness [57].

\section{Grading of evidence}

All members of the Summit agreed that there was welldesigned evidence regarding the statement. Seventy-eight percent voted that the evidence was Category I while 22\% indicated that it was Category II (Table 2).

Level of support

There was unanimous acceptance of the statement among the Summit panelists, although none completely accepted it. Seventy-eight percent expressed some reservations, whereas $22 \%$ had major reservations. In contrast, $19 \%$ of survey participants rejected the statement. Voting details can be found in Fig. 2.

\section{Discussion}

The level of support for the statement among the Summit panelists paralleled the grading of the evidence, suggesting 
the major reservation by $22 \%$ was associated with the perception of a lesser quality of available evidence. Although there was a randomized controlled study (RCT) with results that supported the validity of the statement, the presence of confounding factors in that study (i.e, significantly lower baseline serum IGF-I levels in the pretreatment group) [84] was considered by Summit members to have portended an enhanced subsequent control rate in that group.

Another point expressed by Summit participants related to their voting results was that the statement did not differentiate between microadenomas and macroadenomas. Participants tended to agree that while the evidence supported the use of somatostatin analog therapy among patients with macroadenomas and destined for surgery, the recommendation to pretreat microadenomas was not well supported by the published results.

Since the most robust study offering evidence in support of the value of pretreatment with somatostatin analogs was published only months before the survey, it is possible that a lack of awareness of that RCT may have influenced the voting by survey participants.

\section{Future direction}

Clearly, more studies are required to support the role of therapy with somatostatin analogs among patients destined for surgery. Pretreatment use of somatostatin analogs has been examined largely in nonrandomized studies with several methodological shortcomings. Future studies need to specifically address the use of the products that are currently available, particularly octreotide LAR and lanreotide depot, since the results obtained with these longacting preparations may differ from those observed with the older shorter-acting preparations. Furthermore, the studies should include a sufficient follow-up period to monitor for true long-term outcomes, as well as be better controlled for the respective surgeon, biochemical assay variation, and methods of tumor volume determination.

Until then, the evidence suggests that use of somatostatin analogs prior to transsphenoidal surgery is of value, particularly in patients harboring macroadenomas.

\section{Statement 5: the dosing interval of a somatostatin analog can be extended beyond once every 4 weeks without compromising disease control in patients with acromegaly}

Rationale and definition of statement

Extending the dosing interval of every four week preparations of somatostatin analogs offers the advantage of fewer injections, benefiting the patient with less discomfort and inconvenience and cost reduction.

\section{Literature search}

In order to examine the empirical evidence available to support or refute this statement, a literature search was conducted in October 2008 via PubMed using search terms detailed in Table 3. After review, 7 articles were determined to be pertinent to the statement. One additional article that was published in December 2008 was identified after completion of the search.

\section{Evidence}

All 8 of the studies found to be pertinent to the statement were prospective and open-label, and specifically addressed the issue of extended dosing with various somatostatin analogs in patients with acromegaly. All assessed disease control by monitoring either biochemical response or symptom control or both. Most involved fewer than 30 patients.

Jenkins et al. noted that GH and IGF-I control was maintained in some patients even at 6 weeks after the final dose of octreotide LAR. Mean GH was $<5 \mathrm{mU} / \mathrm{l}$ in 13 and 12 out of 18 patients at 4 and 6 weeks, respectively. IGF-I was normalized in 12 and 11 out of 18 patients, respectively, suggesting that octreotide LAR dosing intervals could be extended to 6 weeks in some patients [58].

Biermasz et al. studied the effectiveness of octreotide LAR given at 6-week intervals in a cohort of 14 patients with active acromegaly who were already controlled (defined as $\mathrm{GH}<5 \mathrm{mU} / 1$ and normal IGF-I) while receiving octreotide LAR every 4 weeks for at least 3 months. During a withdrawal period, mean $\mathrm{GH}$ increased from $1.68 \mathrm{mU} / \mathrm{l}$ at 4 weeks, $2.57 \mathrm{mU} / \mathrm{l}$ at 6 weeks, and $2.89 \mathrm{mU} / \mathrm{l}$ at 8 weeks after administration $(P=0.04,4$ vs. 6 weeks; and $P<$ $0.001,4$ vs. 8 weeks). The mean $\mathrm{GH}$ level was $<5 \mathrm{mU} / \mathrm{l}$ in all patients at all time points, except in one patient at 8 weeks. IGF-I levels also remained normalized in all

Table 3 Literature search strategy

\begin{tabular}{lll}
\hline Search & Textwords & \# of articles \\
\hline Search 1 & $\begin{array}{c}\text { Somatostatin analogs or lanreotide or } \\
\text { octreotide }\end{array}$ & 9615 \\
Search 2 & $\begin{array}{c}\text { Drug administration schedule } \\
\text { or interval }\end{array}$ & 276,345 \\
Search 3 & $\begin{array}{l}\text { Acromegaly } \\
\text { Combine above } \\
\text { Search 4 }\end{array}$ & 7404 \\
Search 5 & $\begin{array}{c}\text { Limith "and" } \\
\text { Search 4 (English and Human) }\end{array}$ & 88 \\
&
\end{tabular}


patients. There was no significant change in symptom or QOL scores. Mean drug level decreased from $1610 \mathrm{ng} / \mathrm{l}$ to $1045 \mathrm{ng} / \mathrm{l}$ and $559 \mathrm{ng} / \mathrm{l}$ at 2, 6, and 8 weeks, respectively [59].

Following the 8-week withdrawal period of the study, patients resumed their previous dose of octreotide LAR, but the drug was administered every 6 weeks. Mean GH remained $<5 \mathrm{mU} / \mathrm{l}$ in 13 out of 14 patients at 26 weeks, and in 13 out of 13 patients at 44 weeks. IGF-I was normal in 11 out of 14 patients at 26 weeks, and in 9 out of 13 patients at 44 weeks. Mean serum octreotide concentration did not change significantly between weeks 6 and 26 or weeks 6 and 44. Mean QOL score decreased from 5 to 4 , and mean symptom score did not change at all. The authors concluded that octreotide LAR dosing intervals can be extended to 6 weeks in some patients, but that careful monitoring (clinical and biochemical) is warranted [59].

Dosing intervals of octreotide LAR were adjusted in Turner's study with 22 patients, based upon mean GH levels of $<5 \mathrm{mU} / \mathrm{l}$. The dosing interval was successfully increased beyond 4 weeks in 20 out of 22 patients; 6 patients were extended to 8 weeks, and 3 patients to 12 weeks. Despite the success with increased dosing intervals in some patients, there was no relationship between the ability to increase intervals and pretreatment mean GH or IGF-I, age, or previous treatments, limiting the ability to predict which patients could successfully receive octreotide LAR at longer dosing intervals [60].

Van Thiel et al. reported the duration of action of lanreotide depot, an every 4 week preparation. Seven patients with active acromegaly who were already receiving octreotide LAR (average duration 2.8 years) with good response were crossed over to lanreotide depot and the dose was titrated to fasting $\mathrm{GH}<5 \mathrm{mU} / \mathrm{l}$ and IGF agematched normal. Although the dosing intervals were not extended with either octreotide LAR or lanreotide depot, GH and IGF-I control was evaluated after a 6-week withdrawal period following at least 1 year of treatment with each drug. While 3 out of 7 patients in each treatment period achieved control of both GH and IGF-I at 4 weeks, only one in each treatment period remained controlled at 6 weeks [61].

A cross-over study by Lucas et al. later examined various fixed dosing intervals of the same $120 \mathrm{mg}$ dose of lanreotide depot in 98 patients who had already been demonstrated to be responsive to somatostatin analogs. The patients were first treated with lanreotide microparticles (LAN MP) $30 \mathrm{mg}$, with the dose interval initially being adjusted to optimally control their disease. The intervals were kept constant for at least the 2 months prior to being switched over to lanreotide depot. The total monthly dose was maintained and the dosing interval was determined by the LAN MP dosing interval. Lanreotide depot intervals were 4,6 , or 8 weeks if the LAN MP intervals had been 7,10 , or 14 days, respectively. $\mathrm{GH}<2.5 \mathrm{ng} / \mathrm{ml}$ were observed with lanreotide depot in $54 \%$ of patients and in $46 \%$ of patients while on LAN MP. Symptoms were also better controlled with lanreotide depot at 4 to 8 week intervals compared with LAN MP at 7 to 14 day intervals [62].

Abrams et al. reported on a study examining different dosing intervals of lanreotide depot in 21 patients who had been receiving $60 \mathrm{mg}, 90 \mathrm{mg}$, or $120 \mathrm{mg}$ at 4-week intervals. Intervals were extended to 6 weeks among patients well controlled on their fixed dose $(n=9)$. Those poorly controlled on a fixed dose every 4 weeks either underwent a dose increase or an interval decrease if they were already at the maximum dose $(120 \mathrm{mg})$. Patients with reasonably good control with their previous fixed dose (defined as IGF-I normal and GH between 1.7 and $2.5 \mu \mathrm{g} / \mathrm{l}$ ) underwent both a $30 \mathrm{mg}$ dose increase and an interval increase to 6 weeks. 7 out of 9 patients who were in good control at baseline remained so at 36 weeks. In contrast, only 1 of 12 patients who were not previously in good control had normalized IGF-I and $\mathrm{GH}$ at 36 weeks, despite dosing intervals of 3 weeks [63].

Finally, Ronchi et al. studied 23 patients who had previously received octreotide LAR every 4 weeks with a $\mathrm{GH}$ reduction at least $50 \%$ of pretreatment values. After a 3-month washout period, lanreotide depot $120 \mathrm{mg}$ was administered every 6 weeks for 4 cycles (treatment period 1). Dosing intervals based on $\mathrm{GH}$ level responses were then increased (6 patients), decreased (12 patients), or maintained (6 patients) for an additional 2 to 3 cycles. The mean biochemical levels and number of well-controlled patients were similar at the end of octreotide LAR treatment and each of the treatment periods, suggesting that extended dosing intervals of lanreotide depot can be extended beyond 4 weeks in approximately half of the patients [64].

A recent study by Colao et al. examined biochemical control and tumor shrinkage associated with lanreotide depot treatment in 26 patients with newly diagnosed acromegaly. Initial dose intervals of lanreotide depot $120 \mathrm{mg}$ were increased from 4 weeks to 6 or 8 weeks, as permitted by maintained control. The final dosage interval was 4 weeks in $35 \%$ of patients; 6 weeks in $31 \%$; and 8 weeks in another $35 \%$. At 12 months, both GH and IGF-I levels were controlled in 54\%. Mean tumor volume decreased from $1405 \mathrm{~mm}^{3}$ at baseline to $960 \mathrm{~mm}^{3}$ at 6 months, and $799 \mathrm{~mm}^{3}$ at 12 months $(P<0.0001)$. Tumor shrinkage $\geq 25 \%$ was experienced by $77 \%$ patients during the 12-month treatment period. Symptoms (hyperhidrosis, paresthesia, and extremity arthralgias) were 
significantly decreased compared with baseline $(P<$ $0.0001, P=0.018$ and $P=0.004$, respectively) [65].

\section{Grading of evidence}

After reviewing the evidence available on this subject, $77 \%$ of the Summit panel indicated that they thought the evidence presented was either Category II or III. Eleven percent thought that the evidence was at Category I and another $11 \%$ indicated that there was not sufficient evidence to form an opinion (Table 2).

\section{Level of support}

In agreement with the Summit panel, only a small percentage of the survey participants completely accepted that the dosing interval of an somatostatin analog can be extended beyond once every 4 weeks without compromising disease control in patients with acromegaly based on available data in the literature. However, the majority of both the survey respondents and Summit members indicated that they accepted the statement with some (50 and $56 \%$, respectively) or major ( $22 \%$ for both groups) reservations. $24 \%$ of the surveyed practitioners rejected the statement while only $11 \%$ of the Summit participants did so (Fig. 2).

\section{Discussion}

There is clear evidence that somatostatin analogs are consistently effective in suppressing mean GH and IGF-I levels in patients with acromegaly and that in selected patients, these agents can be extended beyond the 4-week recommended dosing interval without compromising $\mathrm{GH}$ or IGF-I levels or clinical response. In the subset of responders, the benefit of extended dosing demonstrated both improved cost outcomes and greater acceptance by patients. However, the evidence available to routinely recommend extended dosing is incomplete.

\section{Future direction}

What is unknown is whether there are specific characteristics that might predict a given patient's likelihood of responding to an extended dosing strategy. Future research should address variables that may assist clinicians in predicting those patients most likely to benefit from extended dosing. Studies should also include larger samples and randomized designs. Moreover, the inclusion of patient symptomatology, QOL, and satisfaction outcomes is essential in providing clinicians with comprehensive information on which to base fully informed decisions in patients with acromegaly. Finally, economic analyses would also be of value.

\section{Statement 6: a monthly injection of the depot form of lanreotide or octreotide results in consistent control of hormone levels and symptoms in patients with carcinoid syndrome}

Rationale and definition of statement

Completely effective treatment of carcinoid tumors remains somewhat elusive. It is therefore appropriate to carefully examine this broader topic as we investigate evidence on the statement above. The statement initiates several points of interest-the use of somatostatin analogs, the level of both symptom and hormonal control, as well as the consistency of this control-requiring some review.

Evaluation of the effects of any treatment to control symptoms should at least include the major symptom complex and, if possible, those less frequently recognized. The symptom complex of carcinoid tumors includes flushing in $94 \%$; diarrhea in $78 \%$; cardiac-related complications in $37 \%$; abdominal cramps in $51 \%$; telangiectasia in $25 \%$; bronchoconstriction, edema, and cyanosis in 17$18 \%$; and arthritis, proximal myopathy, and pigmentation in about 7\% [66]. A recent retrospective analysis of 392 patients also found hypertension in $45.9 \%$ and diabetes in $9.7 \%$ of patients.

Serotonin and its excretory products are thought to be the predominant biochemical markers of carcinoid syndrome, but they are notoriously weak means of detecting and monitoring foregut and hindgut carcinoids. Chromogranin $(\mathrm{CgA})$ is possibly the best marker, but levels may not correspond to symptom control. Furthermore, false elevations are known to occur in people with severe hypertension or renal insufficiency and those using proton pump inhibitors [66-68].

Pancreastatin and neurokinin A are two emerging biomarkers that reflect patient prognosis. Both have been found to be independent prognostic indicators and strongly associated with outcome [69-71].

Pancreastatin is a post-translational processing product of $\mathrm{CgA}$ and is known to correlate with the number of liver metastases. It has been found to be an independent indicator $(P<0.001)$ of poor outcome when pretreatment plasma concentration was $>500 \mathrm{pmol} / 1$ in patients with NETs on multivariate, but not univariate analysis [70]. Paradoxically increasing pancreastatin levels during somatostatin analog therapy were observed in some patients and were found to be associated with poorer survival on both univariate and multivariate analyses (both $P<0.001$ ) [70]. In another study, pancreastatin $>5000 \mathrm{pg} / \mathrm{ml}$ pretreatment was associated with 
increased peri-procedure mortality in patients who underwent hepatic artery chemoembolization and also correlated with decreased survival (relative risk [RR] 2.6 [95\% CI, 1.3-5.0]) [69].

A perfect evaluation of symptom and biochemical control would embrace all of these. Unfortunately, regardless of monitoring techniques, there has been a lack of change in long-term outcomes (including survival) from 1973-2002 per a report from Modlin et al., despite the introduction of somatostatin analogs during that time [72]. In light of these observations, the focus of this report is to examine evidence on the ability of somatostatin analogs to control symptoms and biochemistries when given as shortacting or long-acting depot analogs.

Literature search

A PubMed literature search was carried out in October 2008. Search terms used and the number of articles found (total $=1956)$ are as follows:

Lanreotide (542);

Lanreotide AND ATG (6);

Octreotide (6370);

Octreotide LAR (265);

Somatostatin analogue (1324);

Long acting somatostatin analogue (3);

Lanreotide AND octreotide LAR (62);

Lanreotide ATG AND octreotide LAR (4);

Lanreotide AND octreotide LAR AND carcinoid (10);

Somatostatin analogue AND carcinoid (152);

Somatostatin analogue AND neuroendocrine tumor (316);

Long acting somatostatin analogue AND carcinoid (1);

Long acting somatostatin analogue AND neuroendocrine tumor (1); and,

Relevant to statement (6).

A thorough examination yielded only 5 articles both valuable and insightful.

\section{Evidence}

Efficacy of octreotide LAR was demonstrated in a retrospective analysis of 392 patients with carcinoid tumors treated $\geq 4$ months in unpublished data by Anthony and Vinik. The 255 patients with diarrhea at baseline decreased to 93 at 3 months and 47 at 12 months. Flushing was found in 134 patients, decreasing to 52 at 3 months and 29 at 12 months. Heart disease and bronchoconstriction were found in 20 and 21 patients respectively; at 3 months they had decreased to 4 , and at 12 months to 2 , in both conditions. Of particular note was the increasing improvement observed between 3 and 12 months, although as many as
$40-60 \%$ of patients had persistent symptoms. However, just as in Modlin's report, there was no real change in the course of the tumors. Comorbidities such as hyperglycemia $(8.7 \%)$, cholelithiasis $(6.4 \%)$, cholecystitis $(2.8 \%)$, steatorrhea $(2.3 \%)$, and hypoglycemia $(1.5 \%)$ were observed. Unfortunately, the study did not include data on rates of escape from symptom control, use of rescue medication, or the control of biochemical markers as measures of responsiveness.

A prospective multicenter study of 93 patients with preexisting control on octreotide subcutaneous (SC), was one of the earliest papers on the efficacy of octreotide LAR in carcinoid syndrome. After a washout period, octreotide LAR efficacy was compared with that of octreotide SC. Octreotide SC produced complete or partial success in $58.3 \%$ patients whereas the response to octreotide LAR was somewhat dosedependent $(66.7 \%$ with $10 \mathrm{mg} ; 71.4 \%$ with $20 \mathrm{mg}$; and $61.9 \%$ with $30 \mathrm{mg}$ ), although rescue medication was required in $40-60 \%$ cases [73].This has raised a question regarding bioavailability of octreotide in the LAR preparation.

Woltering et al., in a retrospective chart review [74], report that plasma octreotide levels after $30,60,120 \mathrm{mg} /$ month octreotide LAR fell with time and as a result, might not have been reaching receptor saturation levels. While this may partially account for the relative lack of efficacy of octreotide LAR in controlling symptoms, there are other possible explanations. Any "position statement" on symptom control warrants cautious interpretation.

A pharmacokinetic comparison showed that lanreotide depot reaches maximum plasma concentration sooner (mean 1.1 [range $0.25-8$ days] and 2.4 days [range 0.13 18 days], depending on dose) compared with approximately 2 weeks (mean 12.6 [range 0.02-28 days] and 22 days [range 12-34 days]) with octreotide LAR. These differences demonstrate a potential need for using a shortacting analog to control symptoms in the first 2 weeks of initiating octreotide LAR therapy, which was not evident with lanreotide depot [75].

In the first report on efficacy of lanreotide, [76] which was a prospective, open-label, multicenter trial, 39 patients with nonresectable carcinoid syndrome were given lanreotide SR $30 \mathrm{mg}$ IM every 14 days. There was a decrease in the number of flushing and diarrhea episodes after 7 and 15 days of treatment. After 7 days, complete resolution of flushing was seen in $45 \%$ and diarrhea in $17 \%$ of patients. By 30 days, complete resolution was seen in 39 and $30 \%$ of patients, respectively. There was a $>50 \%$ decrease in 5 HIAA (hydroxyindolacetic acid) urinary concentrations in $35 \%$ of patients; no change in $50 \%$; and an increase in $15 \%$ within 7 days. By 30 days, these figures were 18, 57, and $25 \%$, respectively, again emphasizing that long-acting analogs are not impressive when it comes to controlling major symptoms of carcinoid tumors. 
A prospective, open, randomized trial with 33 symptomatic carcinoid syndrome patients compared octreotide 200-300 $\mu \mathrm{g}$ SC 2-3 x/day with lanreotide $30 \mathrm{mg}$ IM every 10 days as 30 -day cross-over treatment periods. Lanreotide and octreotide were equally efficacious in symptom control: flushing 54 vs. $68 \%$ (disappearance or improvement); diarrhea 45 vs. $50 \%$; and abdominal cramps 14 vs. $29 \%$, respectively. There was similar reduction in tumor markers, but lanreotide SR was better tolerated than octreotide $\mathrm{SC}$ due to the difference in injections. Patients preferred lanreotide SR $(68 \%)$ to octreotide SC $(32 \%)(P=0.03)$ [77].

To prospectively assess efficacy and tolerability of lanreotide SR in previously untreated and octreotide LAR treated patients, Ricci et al. examined 25 patients with advanced metastatic NETs, measuring 5-hydroxyindole acetic acid (5-HIAA), plasma CgA, serotonin, calcitonin, and gastrin. Lanreotide SR (30 mg every 14 days [median duration 10 months]) showed significant efficacy in terms of objective RR and biochemical and symptom control among both pretreated and untreated patients. Despite lanreotide SR treatment, disease progressed in 54\% of patients, as did biochemical control in $47.5 \%$ and symptoms in $30 \%$. They were stabilized in only 40,10 , and $5 \%$, respectively [78].

Ricci et al. examined the efficacy of octreotide LAR (20 mg IM every 4 weeks) in 15 patients with metastatic NET with disease progression after lanreotide SR (30 mg every 14 days) induced partial response (PR) or stable disease (SD). Complete responses were observed in 33 and $67 \%$ of patients based on biochemical and symptomatic criteria. Partial responses for tumor growth, biochemistry, and symptom control were noted in 7,8 , and $8 \%$ of patients, respectively. Stable disease was achieved in 40, 33 , and $25 \%$, respectively. Progression occurred in 53 and $26 \%$ of patients as defined by objective and biochemical measures [79].

Finally, Bajetta et al. carried out an open, prospective, randomized 18-week trial in 60 patients with carcinoid tumors. Patients were given either lanreotide depot $120 \mathrm{mg}$ every 6 weeks or lanreotide SR $60 \mathrm{mg}$ every 3 weeks to compare equivalence and measured symptoms, biochemistry, and QOL. Lanreotide depot was as effective as lanreotide SR in controlling NET symptoms, tumor, and QOL [80]. Quality of life was evaluated using the European Organization for Research and Treatment cancer QOL (EORTC QLQ-C30) questionnaire, which has symptom, function, and global health scales. No treatment-related differences were found in any of these domains. Somewhat disappointingly, no effects on these domains were found when comparing entry scores and last observations evaluated 4 to 8 weeks after commencing treatment.
Contrasting with these somewhat disappointing results on tumor growth and hormone response is the recent report on a prospective multicenter study of 15 patients with gastric carcinoid tumors type 1 (GCA1). Here, 14 patients were treated with octreotide LAR and 1 patient with lanreotide depot following excision of the "dominant" lesion during the first gastroscopy. In all patients, size and number of tumors decreased after 6 months of treatment $(P<0.05)$. Serum gastrin levels decreased from mean $898 \pm 418 \mathrm{mU} / \mathrm{l}$ to $304 \pm 278 \mathrm{mU} / \mathrm{l}$ (normal range 40 $108 \mathrm{mU} / 1 ; P<0.005)$. Serum $\mathrm{CgA}$, evaluated in 5 patients, decreased from $370 \pm 183 \mathrm{ng} / \mathrm{ml}$ to $148 \pm 69.3 \mathrm{ng} / \mathrm{ml}$ (mean $\pm \mathrm{SD}$; normal range $19.4-98.1 \mathrm{ng} / \mathrm{ml}, P<0.005$ ) [81]. Thus, somatostatin analogs may be an effective medical therapy in gastric carcinoids, reducing tumor load, decreasing gastrin levels, and exerting an antiproliferation effect on enterochromaffin-like (ECL) cells.

The apparent effectiveness in GCAI gastric carcinoids addresses the issue of the selectivity of somatostatin ana$\operatorname{logs}$ for SST receptors on different tumors. Octreotide and lanreotide bind most strongly to somatostatin receptor-2 (SSTR2), to a lesser extent SSTR3 and SSTR5, and virtually not at all to SSTR1 and SSTR4 [82]. Thus, somatostatin analogs with other receptor specificities may have different effects on selective types of NETs.

\section{Grading of evidence}

Eighty-nine percent of the Summit participants considered the evidence to be Category II. Only 11 percent considered the strongest evidence available was that obtained from case series, reports, or flawed clinical trials (Category III) (Table 2).

\section{Level of support}

Very similar percentages of survey respondents and Summit participants accepted this statement (89 and 88\%, respectively). In both groups, there were a considerable number who had some reservations as well as those with major reservations. Eleven percent of each group rejected the statement, also mostly with reservation. See Fig. 2 for more detail.

\section{Discussion}

Reservation in the acceptance of this statement, similar among both surveyed practitioners and Summit participants, might be related to the presence of multiple components of the statement itself. The level of evidence varied in regard to each of these components (monthly dosing, hormone and symptom control, and consistency of control). 
There is likely little doubt in practitioners' minds that somatostatin analogs are capable of controlling the symptom complex of NETs, but lesser acceptance that they are able to control tumor growth or biochemical abnormalities. In this regard, the quality of evidence is only Category II although what data is available does suggest that they are relatively ineffective; one trial using a poor tool suggests that they may not enhance QOL. Of note, data from a randomized, double blind, placebo-controlled study of 85 patients with gastroenteropancreatic neuroendocrine tumors (the PROMID Study Group) [83] was presented subsequent to the Summit. The octreotide LAR group experienced significantly increased time to progression compared with the placebo group (14.3 vs. 6 months, $P=0.000072$ ). At 6 months, stable disease was noted among 67 and 37\%, respectively [83].

There is overwhelming evidence based on clinical trials, case reports, and case control studies indicating that somatostatin analogs are safe and have the ability to control certain symptoms of carcinoid tumors. Nonetheless, the trials have been relatively small and of short duration. Numerous small studies make it clear that octreotide LAR can control symptoms among approximately two-thirds of subjects, but that escape occurs at varying intervals of time for flushing and diarrhea, requiring the use of short-acting analogs that seem to have greater efficacy. The reason for this discrepancy is not clear and may relate to a variety of reasons such as bioavailability, pharmacokinetics and dynamics, or even changes in the bioavailability of different batches of the drugs.

A paradox is seen regarding response to lanreotide in patients failing octreotide and vice versa. Although the binding to the SSTR of each agent appears to be the same in vitro, these peptides differ sufficiently so that their action in vivo appears to defy the SSTR binding rules. Accruing evidence reveals that loss of bioavailability of octreotide LAR needs to be monitored using blood levels. Escape from control may not reflect loss of efficacy of the drug, tachyphylaxis, appearance of antibodies, or mutation in the cell type of the tumor, but simply a state of decreasing bioavailability. Furthermore, the vast symptom complex and its potential multitude of mechanisms, many of which are not susceptible to inhibition by SST alone, may require combinations with agents targeting the alternate pathogenetic mechanisms.

\section{Future direction}

Better trials are needed to compare the analogs. Head-tohead, randomized comparisons are needed with the different long-acting analogs outlining a clear definition of the patient population. A placebo-controlled trial, using different somatostatin analogs with different specificities for
SSTR and carried out among well-characterized patients with NETs for a sufficient period of time, should be aimed at establishing an appropriate dose range that achieves blood levels optimally saturating SSTRs.

In this day of evidenced-based medicine, orphan diseases such as NETs may have great difficulty fulfilling the requirements of a double-blind, randomized, placebo-controlled trial in a sufficiently large population of subjects. Since there are no centers with a sufficiently large population to carry out these needed studies, it behooves NEToriented researchers to pool resources. In this way, larger scale multicenter studies may eventually yield rewarding results. Without this cooperative effort, our expectation can probably never exceed a Category II recommendation or acceptance.

Determining the reasons for differences in response may require evaluation of pharmacokinetics and pharmacodynamics of different long-acting analogs compared with their short-lived counterparts. In addition, the presence of different receptor subtypes and their specificity in different NET tumor types might dictate a need for tailoring somatostatin analogs to the tumor.

The disappointing results with somatostatin analogs on tumor growth over the last 3 decades should spur investigators into seeking new and alternate approaches to controlling tumor growth, such as tyrosine kinase inhibitors [84], mammalian target of rapamycin (mTOR) inhibitors [85], or combination therapies that include interferons [86].

Additionally, many studies have focused upon diarrhea and flushing, but ignored the remainder of the vast symptom complex of NETs. Indeed, none have looked into the effects of cardiovascular disease, bronchospasm, or bone metastases. Since these are slow-growing tumors with a relatively long period of stable growth and tumor mass not readily amenable to treatment, QOL measures are needed to address all symptoms that may beset the patient with an NET [87].

\section{Conclusions}

An endocrinology panel was convened to provide evidence-based recommendations regarding the use of $\mathrm{GH}$ and IGF-I measurements in acromegaly and the use of somatostatin analogs in the management of patients with acromegaly or NETs. Overall, evidence supported the use of IGF-I measurements to assess disease control in acromegaly and the need for standardized assays and large normative data bases was emphasized. Summit panel opinion, as compared to surveyed community-based practitioners, is detailed in Fig. 2.

Panelists felt that data regarding either random $\mathrm{GH}$ levels or GH day curves in assessing disease control and 
mortality were well supported by the literature. However, the role of GH suppression as a therapeutic endpoint in the care of patients with known acromegaly was not substantiated. The change in assay methodology over the years and the increased sensitivity of newer GH assays compared to older assays was noted. The recommended goal of therapy for patients with acromegaly is $\mathrm{GH}<1 \mu \mathrm{g} / \mathrm{l}$ because a $<2.5 \mu \mathrm{g} / \mathrm{l}$ (as assessed by older assays in past studies) correlated with normalization of the high mortality rate associated with acromegaly. The Summit panel concluded that both IGF-I and GH levels could be used to evaluate the activity of acromegaly, particularly when values were discrepant. However, GH cutoff values should be interpreted and reanalyzed according to patient's gender, age, BMI, gonadal status, and specific assay used.

Regarding the use of somatostatin analogs in management of acromegaly, consideration was given to the statements that patients could be controlled with prolonged dosing intervals. Based on the small number of studies available, none controlled, the Summit panel felt that although some patients could be controlled with prolonged dosing intervals, there was insufficient data to recommend this and no data available as to predictors of this response.

As for the use of somatostatin analogs prior to surgery in patients with acromegaly, the Summit panel concluded that the administration of somatostatin analogs prior to transsphenoidal surgery improves surgical outcome and disease morbidity associated with acromegaly. However, these data applied to macroadenomas and there was no evidence supporting its use in microadenomas unless metabolic or anesthesia risks associated with soft tissue swelling were of immediate concern. Longer term randomized studies were needed to assess the overall benefit of this strategy.

Finally, the Summit panel considered the use of somatostatin analogs in the management of NETs. There was a consensus that this therapy can control a number of symptoms due to carcinoid tumors, although studies have been of short duration. However, in contrast to the data in acromegaly, escape may occur, requiring the use of shortacting analogs. This escape may reflect tachyphylaxis, development of antibodies, changing cell populations in the tumor, and/or decreasing bioavailability. In addition, although somatostatin analogs can control disease symptoms, hormone hypersecretion, and tumor growth in many patients with acromegaly, these analogs are not effective in treating tumor burden in NETs.

Open Access This article is distributed under the terms of the Creative Commons Attribution Noncommercial License which permits any noncommercial use, distribution, and reproduction in any medium, provided the original author(s) and source are credited.

\section{References}

1. Vierhapper H, Heinze G, Gessl A, Exner M, Bieglmayr C (2003) Use of the oral glucose tolerance test to define remission in acromegaly. Metabolism 52:181-185

2. Ronchi CL, Varca V, Giavoli C et al (2005) Long-term evaluation of postoperative acromegalic patients in remission with previous and newly proposed criteria. J Clin Endocrinol Metab 90:1377-1382

3. Nomikos P, Buchfelder M, Fahlbusch R (2005) The outcome of surgery in 668 patients with acromegaly using current criteria of biochemical 'cure'. Eur J Endocrinol 152:379-387

4. Jenkins PJ, Bates P, Carson MN, Stewart PM, Wass JA (2006) Conventional pituitary irradiation is effective in lowering serum growth hormone and insulin-like growth factor-I in patients with acromegaly. J Clin Endocrinol Metab 91:12391245

5. Clemmons DR, Van Wyk JJ, Ridgway EC, Kliman B, Kjellberg RN, Underwood LE (1979) Evaluation of acromegaly by radioimmunoassay of somatomedin-C. N Engl J Med 301: $1138-1142$

6. Puder JJ, Nilavar S, Post KD, Freda PU (2005) Relationship between disease-related morbidity and biochemical markers of activity in patients with acromegaly. J Clin Endocrinol Metab 90:1972-1978

7. Dimaraki EV, Jaffe CA, DeMott-Friberg R, Chandler WF, Barkan AL (2002) Acromegaly with apparently normal GH secretion: implications for diagnosis and follow-up. J Clin Endocrinol Metab 87:3537-3542

8. Pivonello R, Galderisi M, Auriemma RS et al (2007) Treatment with growth hormone receptor antagonist in acromegaly: effect on cardiac structure and performance. J Clin Endocrinol Metab 92:476-482

9. Colao A, Pivonello R, Auriemma RS et al (2006) Predictors of tumor shrinkage after primary therapy with somatostatin analogs in acromegaly: a prospective study in 99 patients. J Clin Endocrinol Metab 91:2112-2118

10. Alexopoulou O, Bex M, Abs R, T'Sjoen G, Velkeniers B, Maiter D (2008) Divergence between growth hormone and insulin-like growth factor-I concentrations in the follow-up of acromegaly. J Clin Endocrinol Metab 93:1324-1330

11. Paisley AN, Rowles SV, Roberts ME et al (2007) Treatment of acromegaly improves quality of life, measured by AcroQol. Clin Endocrinol (Oxf) 67:358-362

12. Kauppinen-Makelin R, Sane T, Sintonen $\mathrm{H}$ et al (2006) Quality of life in treated patients with acromegaly. J Clin Endocrinol Metab 91:3891-3896

13. Trepp R, Everts R, Stettler C et al (2005) Assessment of quality of life in patients with uncontrolled vs. controlled acromegaly using the acromegaly quality of life questionnaire (AcroQoL). Clin Endocrinol (Oxf) 63:103-110

14. Holdaway IM, Rajasoorya RC, Gamble GD (2004) Factors influencing mortality in acromegaly. J Clin Endocrinol Metab 89:667-674

15. Holdaway IM, Bolland MJ, Gamble GD (2008) A meta-analysis of the effect of lowering serum levels of GH and IGF-I on mortality in acromegaly. Eur J Endocrinol 159:89-95

16. Massart C, Poirier JY (2006) Serum insulin-like growth factor-I measurement in the follow-up of treated acromegaly: comparison of four immunoassays. Clin Chim Acta 373:176-179

17. Alexander L, Appleton D, Hall R, Ross WM, Wilkinson R (1980) Epidemiology of acromegaly in the Newcastle region. Clin Endocrinol (Oxf) 12:71-79

18. Holdaway IM (2007) Excess mortality in acromegaly. Horm Res 68(5):166-172 
19. Kauppinen-Makelin R, Sane T, Reunanen A et al (2005) A nationwide survey of mortality in acromegaly. J Clin Endocrinol Metab 90:4081-4086

20. Bidlingmaier M, Strasburger CJ (2007) Growth hormone assays: current methodologies and their limitations. Pituitary 10:115-119

21. Bates AS, Van't Hoff W, Jones JM, Clayton RN (1993) An audit of outcome of treatment in acromegaly. Q J Med 86:293-299

22. Abosch A, Tyrrell JB, Lamborn KR, Hannegan LT, Applebury CB, Wilson CB (1998) Transsphenoidal microsurgery for growth hormone-secreting pituitary adenomas: initial outcome and longterm results. J Clin Endocrinol Metab 83:3411-3418

23. Orme SM, McNally RJ, Cartwright RA, Belchetz PE (1998) Mortality and cancer incidence in acromegaly: a retrospective cohort study. United Kingdom acromegaly study group. J Clin Endocrinol Metab 83:2730-2734

24. Beauregard C, Truong U, Hardy J, Serri O (2003) Long-term outcome and mortality after transsphenoidal adenomectomy for acromegaly. Clin Endocrinol (Oxf) 58:86-91

25. Mestron A, Webb SM, Astorga R et al (2004) Epidemiology, clinical characteristics, outcome, morbidity and mortality in acromegaly based on the Spanish acromegaly registry (Registro Espanol de Acromegalia, REA). Eur J Endocrinol 151:439-446

26. Trepp R, Stettler C, Zwahlen M, Seiler R, Diem P, Christ ER (2005) Treatment outcomes and mortality of 94 patients with acromegaly. Acta Neurochir (Wien) 147:243-251

27. Arita K, Kurisu K, Tominaga A et al (2003) Mortality in 154 surgically treated patients with acromegaly-a 10-year follow-up survey. Endocr J 50:163-172

28. Biermasz NR, Dekker FW, Pereira AM et al (2004) Determinants of survival in treated acromegaly in a single center: predictive value of serial insulin-like growth factor I measurements. J Clin Endocrinol Metab 89:2789-2796

29. Ayuk J, Clayton RN, Holder G, Sheppard MC, Stewart PM, Bates AS (2004) Growth hormone and pituitary radiotherapy, but not serum insulin-like growth factor-I concentrations, predict excess mortality in patients with acromegaly. J Clin Endocrinol Metab 89:1613-1617

30. Holdaway IM, Rajasoorya CR, Gamble GD, Stewart AW (2003) Long-term treatment outcome in acromegaly. Growth Horm IGF Res 13:185-192

31. Lieberman SA, Hoffman AR (1990) Sequelae to acromegaly: reversibility with treatment of the primary disease. Horm Metab Res 22:313-318

32. Carmichael JD, Bonert VS, Mirocha JM, Melmed S (2008) The utility of oral glucose tolerance testing for diagnosis and assessment of treatment outcomes in 166 patients with acromegaly. J Clin Endocrinol Metab. doi:101210/jc2008-1371

33. Colao A, Pivonello R, Cappabianca P et al (2005) Effect of gender and gonadal status on the long-term response to somatostatin analogue treatment in acromegaly. Clin Endocrinol (Oxf) 63:342-349

34. Parkinson C, Renehan AG, Ryder WD, O'Dwyer ST, Shalet SM, Trainer PJ (2002) Gender and age influence the relationship between serum GH and IGF-I in patients with acromegaly. Clin Endocrinol (Oxf) 57:59-64

35. Parkinson C, Ryder WD, Trainer PJ (2001) The relationship between serum GH and serum IGF-I in acromegaly is genderspecific. J Clin Endocrinol Metab 86:5240-5244

36. Colao A, Pivonello R, Cavallo LM et al (2006) Age changes the diagnostic accuracy of mean profile and nadir growth hormone levels after oral glucose in postoperative patients with acromegaly. Clin Endocrinol (Oxf) 65:250-256

37. Freda PU, Landman RE, Sundeen RE, Post KD (2001) Gender and age in the biochemical assessment of cure of acromegaly. Pituitary 4:163-171
38. Arafat AM, Mohlig M, Weickert MO et al (2008) Growth hormone response during oral glucose tolerance test: the impact of assay method on the estimation of reference values in patients with acromegaly and in healthy controls, and the role of gender, age, and body mass index. J Clin Endocrinol Metab 93:12541262

39. Damjanovic SS, Neskovic AN, Petakov MS et al (2005) Clinical indicators of biochemical remission in acromegaly: does incomplete disease control always mean therapeutic failure? Clin Endocrinol (Oxf) 62:410-417

40. van Thiel SW, Bax JJ, Biermasz NR et al (2005) Persistent diastolic dysfunction despite successful long-term octreotide treatment in acromegaly. Eur J Endocrinol 153:231-238

41. Colao A, Pivonello R, Galderisi M et al (2008) Impact of treating acromegaly first with surgery or somatostatin analogs on cardiomyopathy. J Clin Endocrinol Metab 93:2639-2646

42. Bevan JS, Atkin SL, Atkinson AB et al (2002) Primary medical therapy for acromegaly: an open, prospective, multicenter study of the effects of subcutaneous and intramuscular slow-release octreotide on growth hormone, insulin-like growth factor-I, and tumor size. J Clin Endocrinol Metab 87:4554-4563

43. Melmed S, Sternberg R, Cook D et al (2005) A critical analysis of pituitary tumor shrinkage during primary medical therapy in acromegaly. J Clin Endocrinol Metab 90:4405-4410

44. Baldelli R, Colao A, Razzore P et al (2000) Two-year follow-up of acromegalic patients treated with slow release lanreotide (30 mg). J Clin Endocrinol Metab 85:4099-4103

45. Colao A, Pivonello R, Rosato F et al (2006) First-line octreotideLAR therapy induces tumour shrinkage and controls hormone excess in patients with acromegaly: results from an open, prospective, multicentre trial. Clin Endocrinol (Oxf) 64:342-351

46. Cozzi R, Montini M, Attanasio R et al (2006) Primary treatment of acromegaly with octreotide LAR: a long-term (up to nine years) prospective study of its efficacy in the control of disease activity and tumor shrinkage. J Clin Endocrinol Metab 91:13971403

47. Mercado M, Borges F, Bouterfa $\mathrm{H}$ et al (2007) A prospective, multicentre study to investigate the efficacy, safety and tolerability of octreotide LAR (long-acting repeatable octreotide) in the primary therapy of patients with acromegaly. Clin Endocrinol (Oxf) 66:859-868

48. Biermasz NR, van Dulken H, Roelfsema F (1999) Direct postoperative and follow-up results of transsphenoidal surgery in 19 acromegalic patients pretreated with octreotide compared to those in untreated matched controls. J Clin Endocrinol Metab 84:35513555

49. Kristof RA, Stoffel-Wagner B, Klingmuller D, Schramm J (1999) Does octreotide treatment improve the surgical results of macroadenomas in acromegaly? A randomized study. Acta Neurochir (Wien) 141:399-405

50. Plockinger U, Quabbe HJ (2005) Presurgical octreotide treatment in acromegaly: no improvement of final growth hormone $(\mathrm{GH})$ concentration and pituitary function. A long-term case-control study. Acta Neurochir (Wien) 147:485-493

51. Losa M, Mortini P, Urbaz L, Ribotto P, Castrignano T, Giovanelli M (2006) Presurgical treatment with somatostatin analogs in patients with acromegaly: effects on the remission and complication rates. J Neurosurg 104:899-906

52. Abe T, Ludecke DK (2001) Effects of preoperative octreotide treatment on different subtypes of $90 \mathrm{GH}$-secreting pituitary adenomas and outcome in one surgical centre. Eur J Endocrinol 145:137-145

53. Barkan AL, Lloyd RV, Chandler WF et al (1988) Preoperative treatment of acromegaly with long-acting somatostatin analog SMS 201-995: shrinkage of invasive pituitary macroadenomas 
and improved surgical remission rate. J Clin Endocrinol Metab 67:1040-1048

54. Stevenaert A, Beckers A (1993) Presurgical octreotide treatment in acromegaly. Acta Endocrinol (Copenh) 129(1):18-20

55. Colao A, Ferone D, Cappabianca P et al (1997) Effect of octreotide pretreatment on surgical outcome in acromegaly. J Clin Endocrinol Metab 82:3308-3314

56. Carlsen SM, Lund-Johansen M, Schreiner T et al (2008) Preoperative octreotide treatment in newly diagnosed acromegalic patients with macroadenomas increases cure short-term postoperative rates: a prospective, randomized trial. J Clin Endocrinol Metab 93:2984-2990

57. Murray RD, Melmed S (2008) A critical analysis of clinically available somatostatin analog formulations for therapy of acromegaly. J Clin Endocrinol Metab 93:2957-2968

58. Jenkins PJ, Akker S, Chew SL, Besser GM, Monson JP, Grossman AB (2000) Optimal dosage interval for depot somatostatin analogue therapy in acromegaly requires individual titration. Clin Endocrinol (Oxf) 53:719-724

59. Biermasz NR, van den Oever NC, Frolich M et al (2003) Sandostatin LAR in acromegaly: a 6-week injection interval suppresses GH secretion as effectively as a 4-week interval. Clin Endocrinol (Oxf) 58:288-295

60. Turner HE, Thornton-Jones VA, Wass JA (2004) Systematic dose-extension of octreotide LAR: the importance of individual tailoring of treatment in patients with acromegaly. Clin Endocrinol (Oxf) 61:224-231

61. van Thiel SW, Romijn JA, Biermasz NR et al (2004) Octreotide long-acting repeatable and lanreotide autogel are equally effective in controlling growth hormone secretion in acromegalic patients. Eur J Endocrinol 150:489-495

62. Lucas T, Astorga R (2006) Efficacy of lanreotide autogel administered every 4-8 weeks in patients with acromegaly previously responsive to lanreotide microparticles $30 \mathrm{mg}$ : a phase III trial. Clin Endocrinol (Oxf) 65:320-326

63. Abrams P, Alexopoulou O, Abs R, Maiter D, Verhelst J (2007) Optimalization and cost management of lanreotide-autogel therapy in acromegaly. Eur J Endocrinol 157:571-577

64. Ronchi CL, Boschetti M, Degli Uberti EC et al (2007) Efficacy of a slow-release formulation of lanreotide (autogel) $120 \mathrm{mg}$ ) in patients with acromegaly previously treated with octreotide long acting release (LAR): an open, multicentre longitudinal study. Clin Endocrinol (Oxf) 67:512-519

65. Colao A, Auriemma RS, Rebora A et al (2009) Significant tumour shrinkage after 12 months of lanreotide autogel-120 mg treatment given first-line in acromegaly. Clin Endocrinol (Oxf) $71: 237-245$

66. Vinik AI, O’Dorisio TM, Woltering EA, Go VL (2006) Neuroendocrine tumors: a comprehensive guide to diagnosis and management, 1st edn. Interscience Institute, Inglewood, California, pp 10-16

67. Vinik AI, Silva M (2009) Biochemical testing for neuroendocrine tumors. In: Lloyd RV (ed) Endocrine pathology: differential diagnosis and molecular advances, 2nd edn. Humana Press Inc, Totowa, New Jersey

68. Zatelli MC, Torta M, Leon A et al (2007) Chromogranin A as a marker of neuroendocrine neoplasia: an Italian Multicenter Study. Endocr Relat Cancer 14:473-482

69. Bloomston M, Al-Saif O, Klemanski D et al (2007) Hepatic artery chemoembolization in 122 patients with metastatic carcinoid tumor: lessons learned. J Gastrointest Surg 11:264-271

70. Stronge RL, Turner GB, Johnston BT et al (2008) A rapid rise in circulating pancreastatin in response to somatostatin analogue therapy is associated with poor survival in patients with neuroendocrine tumours. Ann Clin Biochem 45:560-566
71. Turner GB, Johnston BT, McCance DR et al (2006) Circulating markers of prognosis and response to treatment in patients with midgut carcinoid tumours. Gut 55:1586-1591

72. Modlin IM, Champaneria MC, Chan AK, Kidd M (2007) A threedecade analysis of 3, 911 small intestinal neuroendocrine tumors: the rapid pace of no progress. Am J Gastroenterol 102:1464-1473

73. Rubin J, Ajani J, Schirmer W et al (1999) Octreotide acetate long-acting formulation versus open-label subcutaneous octreotide acetate in malignant carcinoid syndrome. J Clin Oncol 17:600-606

74. Woltering EA, Salvo VA, O'Dorisio TM et al (2008) Clinical value of monitoring plasma octreotide levels during chronic octreotide long-acting repeatable therapy in carcinoid patients. Pancreas 37:94-100

75. Astruc B, Marbach P, Bouterfa $\mathrm{H}$ et al (2005) Long-acting octreotide and prolonged-release lanreotide formulations have different pharmacokinetic profiles. J Clin Pharmacol 45:836-844

76. Ruszniewski P, Ducreux M, Chayvialle JA et al (1996) Treatment of the carcinoid syndrome with the longacting somatostatin analogue lanreotide: a prospective study in 39 patients. Gut 39:279-283

77. O'Toole D, Ducreux M, Bommelaer G et al (2000) Treatment of carcinoid syndrome: a prospective crossover evaluation of lanreotide versus octreotide in terms of efficacy, patient acceptability, and tolerance. Cancer 88:770-776

78. Ricci S, Antonuzzo A, Galli L et al (2000) Long-acting depot lanreotide in the treatment of patients with advanced neuroendocrine tumors. Am J Clin Oncol 23:412-415

79. Ricci S, Antonuzzo A, Galli L et al (2000) Octreotide acetate long-acting release in patients with metastatic neuroendocrine tumors pretreated with lanreotide. Ann Oncol 11:1127-1130

80. Bajetta E, Procopio G, Catena L et al (2006) Lanreotide autogel every 6 weeks compared with Lanreotide microparticles every 3 weeks in patients with well differentiated neuroendocrine tumors: a Phase III Study. Cancer 107:2474-2481

81. Grozinsky-Glasberg S, Kaltsas G, Gur C et al (2008) Long-acting somatostatin analogues are an effective treatment for type 1 gastric carcinoid tumours. Eur J Endocrinol 159:475-482

82. Bruns C, Lewis I, Briner U, Meno-Tetang G, Weckbecker G (2002) SOM230: a novel somatostatin peptidomimetic with broad somatotropin release inhibiting factor (SRIF) receptor binding and a unique antisecretory profile. Eur J Endocrinol 146:707-716

83. Arnold R, Muller H, Schade-Brittinger C et al. (2009) Placebocontrolled, double-blind, prospective, randomized study of the effect of octreotide LAR in the control of tumor growth in patients with metastatic neuroendocrine midgut tumors: a report from the PROMID study group [ASCO abstract 4508]. J Clin Oncol 27:abstr 4508

84. Kulke MH, Lenz HJ, Meropol NJ et al (2008) Activity of sunitinib in patients with advanced neuroendocrine tumors. J Clin Oncol 26:3403-3410

85. Yao JC, Phan A, Hoff PM et al (2008) Targeting vascular endothelial growth factor in advanced carcinoid tumor: a random assignment phase II study of depot octreotide with bevacizumab and pegylated interferon alpha-2b. J Clin Oncol 26:1316-1323

86. Modlin IM, Moss SF, Chung DC, Jensen RT, Snyderwine E (2008) Priorities for improving the management of gastroenteropancreatic neuroendocrine tumors. J Natl Cancer Inst 100: 1282-1289

87. Vinik E, Carlton CA, Silva MP, Vinik AI (2009) Development of the Norfolk quality of life tool for assessing patients with neuroendocrine tumors. Pancreas. doi:101097/MPA0b013e31819b 6441 